\title{
AN EXTENSION OF SCHREIER UNCONDITIONALITY
}

\author{
G. ANDROULAKIS, F. SANACORY*
}

\begin{abstract}
The main result of the paper extends the classical result of E. Odell on Schreier unconditionality to arrays in Banach spaces. An application is given on the "multiple of the inclusion plus compact" problem which is further applied to a hereditarily indecomposable Banach space constructed by N. Dew.
\end{abstract}

\section{INTRODUCTION}

A finite subset $F$ of $\mathbb{N}$ is called a Schreier set if $|F| \leq \min (F)$ (where $|F|$ denotes the cardinality of $F$ ). The important notion of Schreier unconditionality was introduced by E. Odell [16] and has inspired rich literature on the subject (see for example [3], [8]). Earlier very similar results can be found in [15, page 77] and [19, Theorem 2.1']. A basic sequence $\left(x_{n}\right)$ in a Banach space is defined to be Schreier unconditional if there is a constant $C>0$ such that for all scalars $\left(a_{i}\right) \in c_{00}$ and for all Schreier sets $F$ we have

$$
\left\|\sum_{i \in F} a_{i} e_{i}\right\| \leq C\left\|\sum a_{i} e_{i}\right\| .
$$

In this case $\left(e_{i}\right)$ is called $C$-Schreier unconditional.

Theorem 1.1. [16] Let $\left(x_{n}\right)$ be a normalized weakly null sequence in a Banach space. Then for any $\varepsilon>0,\left(x_{n}\right)$ contains a $(2+\varepsilon)$-Schreier unconditional subsequence.

Our main result is Theorem 1.2 where we extend Theorem 1.1 to arrays of vectors of a Banach space such that each row is a seminormalized weakly null sequence. Then Theorem 1.2 guarantees the existence of a subarray which preserves all the rows of the original array and has a Schreier type of unconditionality. We now define the notions of array, subarray and regular array in a Banach space. An array in a Banach space $X$ is a sequence of vectors in $\left(x_{i, j}\right)_{i \in \mathbb{N} ; j \in J_{i}} \subseteq X$ where $J_{i}$ is an infinite subsequence of $\mathbb{N}$ for all $i \in \mathbb{N}$, say $J_{i}=\left\{j_{i, 1}<j_{i, 2}<\cdots\right\}$ and $\left(x_{i, j_{i, k}}\right)_{k \in \mathbb{N}}$ is a seminormalized weakly null sequence in $X$ for all $i \in \mathbb{N}$. The reader is warned that the paper [12] also considers arrays of vectors with different properties. Let $<_{r \ell}$ denote the reverse lexicographical order on $\mathbb{N}^{2}$. Let $\left(x_{i, j}\right)_{i \in \mathbb{N} ; j \in J_{i}}$ be an array in a Banach space $X$. A subarray of $\left(x_{i, j}\right)_{i \in \mathbb{N} ; j \in J_{i}}$ is an array $\left(y_{i, \ell}\right)_{i \in \mathbb{N} ; \ell \in L_{i}}$ in $X$ which satisfies the following two properties:

$$
\left\{y_{i, \ell}: \ell \in L_{i}\right\} \subseteq\left\{x_{i, j}: j \in J_{i}\right\} \text { for all } i \in \mathbb{N}
$$

Date: May 15, 2007.

2000 Mathematics Subject Classification. 46A32, 47B07.

Key words and phrases. Schreier unconditionality, compact operators, hereditarily indecomposable Banach space.

*The present paper is part of the Ph.D thesis of the second author who is partially supported under Prof. Girardi's NSF grant DMS-0306750. 
and

if $J_{i}=\left\{j_{i, 1}<j_{i, 2}<\cdots\right\}$, and $L_{i}=\left\{\ell_{i, 1}<\ell_{i, 2}<\cdots\right\}$ for all $i \in \mathbb{N}$ then there

exists a $<_{r \ell}$-order preserving map $H:\left\{\left(i, j_{i, k}\right): i, k \in \mathbb{N}\right\} \rightarrow\left\{\left(i, j_{i, k}\right): i, k \in \mathbb{N}\right\}$ such that $y_{i, \ell_{i, k}}=x_{H\left(i, j_{i, k}\right)}$ for all $i, k \in \mathbb{N}$.

A regular array in a Banach space $X$ is an array $\left(x_{i, j}\right)_{i, j \in \mathbb{N} ; i \leq j}$ which is a basic sequence when it is ordered with the reverse lexicographic order: $x_{1,1}, x_{1,2}, x_{2,2}, x_{1,3}, x_{2,3}, x_{3,3}, x_{1,4}, \ldots$. For convenience, throughout this paper, we denote the index set of a regular array by $I$, i.e. $I=\{(i, j) \in \mathbb{N} \times \mathbb{N}: i \leq j\}$.

Theorem 1.2. Let $\left(x_{i, j}\right)_{(i, j) \in I}$ be an array in a Banach space $X,\left(M_{j}\right)_{j \in \mathbb{N}} \subseteq \mathbb{N}$ be an increasing sequence of integers and $\varepsilon>0$. Then there exists a regular subarray $\left(y_{i, j}\right)_{(i, j) \in I}$ of $\left(x_{i, j}\right)_{(i, j) \in I}$ such that for any finitely supported scalars $\left(a_{i, j}\right)_{(i, j) \in I}, k_{0} \in \mathbb{N}$ and $F \subseteq \mathbb{N}$ with $|F| \leq M_{\min (F)}$ and $k_{0} \leq \min (F)$ we have

$$
\left\|\sum_{(i, j) \in I} a_{i, j} y_{i, j}\right\| \geq \frac{1}{2+\varepsilon}\left\|\sum_{j \in F} a_{k_{0}, j} y_{k_{0}, j}\right\| .
$$

The "multiple of the inclusion plus compact problem" was asked by W.T. Gowers [9] and asks whether for every infinite dimensional Banach space $X$ there exists a closed subspace $Y$ of $X$ and a bounded linear operator from $Y$ to $X$ which is not a compact perturbation of a multiple of the inclusion map from $Y$ to $X$. Note that if a Banach space $X$ contains an unconditional basic sequence $\left(x_{n}\right)_{n}$ then the operator $T \in \mathcal{L}\left(\left[\left(x_{n}\right)_{n}\right], X\right)$ defined by $T\left(x_{n}\right)=(-1)^{n} x_{n}$ does not belong to $\mathbb{C} i_{Y \rightarrow X}+\mathcal{K}(Y, X)$ (where $\mathcal{L}(Y, X)$ and $\mathcal{K}(Y, X)$ denote respectively the space of all (linear bounded) operators and all compact operators from $Y$ to $X$; if $Y$ is a subspace of $X$ then $i_{Y \rightarrow X}$ denotes the inclusion from $Y$ to $X$ ). Thus if a Banach space $X$ contains an unconditional basic sequence then the "multiple of the inclusion plus compact" problem has an affirmative answer for $X$. Hence for the "multiple of the inclusion plus compact" problem we restrict our attention to Banach spaces with no unconditional basic sequences. Recall that by the Gowers' dichotomy [9] every Banach space contains an unconditional basic sequence or a hereditarily indecomposable (HI) subspace. Recall that a Banach space $X$ is called HI if no infinite dimensional closed subspace $Y$ of $X$ contains a complemented subspace $Z$ which is of both infinite dimension and infinite codimension in $Y$ [11]. Therefore for the "multiple of the inclusion plus compact problem" we only examine HI saturated Banach spaces. In Section 3 we prove Theorems 3.2 and 3.8 which give sufficient conditions on a Banach space $X$ so that the "multiple of the inclusion plus compact" problem to have an affirmative answer in $X$, and extend results of [2] and [21]. In his 2000 dissertation N. Dew [7] introduced a new HI space which we refer to as space $D$. In Section 4 we examine some of the basic properties of $D$ and we apply Theorem 3.8 to prove that the "multiple of the inclusion plus compact" problem has an affirmative answer in $D$.

\section{Extension of OdelL's Schreier UnCONditionality}

In this section we will prove our main Theorem, 1.2. We need several remarks and lemmas until we reach its proof. 
Remark 2.1. If $\left(x_{i, j}\right)_{(i, j) \in I}$ is a regular array and $\left(y_{i, j}\right)_{(i, j) \in I}$ is a subarray of $\left(x_{i, j}\right)_{(i, j) \in I}$ then $\left(y_{i, j}\right)_{(i, j) \in I}$ is also regular.

The proof of the following remark can be found in functional analysis text books such as [1, Theorem 1.5.2] or [14, Lemma 1.a.5]).

Remark 2.2. Let $\left(x_{i}\right)_{i=1}^{N}$ be a finite basic sequence in some infinite dimensional Banach space $X$ having basis constant $C$. Let $\left(y_{i}\right)$ be a seminormalized weakly null sequence $X$ and $\varepsilon>0$. Then there exists an $n \in \mathbb{N}$ such that $\left(x_{1}, x_{2}, \ldots, x_{N}, y_{n}\right)$ is a basic sequence with constant $C(1+\varepsilon)$.

By repeated application of Remark 2.2 we obtain the following.

Remark 2.3. Let $X$ be a Banach space and $\left(x_{i, j}\right)_{(i, j) \in I}$ be an array in $X$. Then there exists a subarray $\left(y_{i, j}\right)_{(i, j) \in I}$ of $\left(x_{i, j}\right)_{(i, j) \in I}$ which is regular. Moreover, the basis constant of $\left(y_{i, j}\right)_{(i, j) \in I}$ can be chosen to be arbitrarily close to 1.

For $p \in \mathbb{N}$ any element $\vec{a}=\left(a_{i}\right)_{i=1}^{p}$ of $\mathbb{R}^{p}$ will be called a $p$-pattern and for such $\vec{a}$ define $|\vec{a}|:=p$. Let $\left(x_{i, j}\right)_{(i, j) \in I}$ be a regular array in a Banach space $X$. Let $f \in X^{*}, k \in \mathbb{N}$, $\vec{a}=\left(a_{i}\right)_{i=1}^{p}$ a $p$-pattern and $F=\left\{j_{1}, j_{2} \ldots, j_{p}\right\} \subseteq \mathbb{N}$. We say that $f$ has pattern $\vec{a}$ on $(k, F)$ with respect to $\left(x_{i, j}\right)_{(i, j) \in I}$ if $f\left(x_{k, j_{i}}\right)=a_{i}$ for all $i \in\{1,2, \ldots, p\}$.

Lemma 2.4. Let $\left(x_{i, j}\right)_{(i, j) \in I}$ be a regular array in a Banach space $X, \vec{a}$ be a p-pattern, $\mathcal{F} \subseteq 2 B a\left(X^{*}\right), \delta>0$ and $i_{0}, j_{0}, k_{0} \in \mathbb{N}$ with $j_{0} \geq i_{0}$. Then there exists a subarray $\left(y_{i, j}\right)_{(i, j) \in I}$ of $\left(x_{i, j}\right)_{(i, j) \in I}$ such that for any $F \subseteq\left\{k_{0}, k_{0}+1, k_{0}+2, \ldots\right\}$, with $\left(i_{0}, j_{0}\right)<_{r \ell}\left(k_{0}, \min (F)\right)$ and $|F|=p$ we have the following:

If there exists $f \in \mathcal{F}$ having pattern $\vec{a}$ on $\left(k_{0}, F\right)$ with respect to $\left(y_{i, j}\right)_{(i, j) \in I}$ then there exists $g \in \mathcal{F}$ having pattern $\vec{a}$ on $\left(k_{0}, F\right)$ with respect to $\left(y_{i, j}\right)_{(i, j) \in I}$ and $\left|g\left(y_{i_{0}, j_{0}}\right)\right|<\delta$.

Additionally, $\left(y_{i, j}\right)_{(i, j) \in I}$ can be chosen to satisfy $y_{i, j}=x_{i, j}$ for all $(i, j)<_{r \ell}\left(i_{0}, j_{0}\right)$.

Proof. First note that there exists $m \in \mathbb{N}$ such that for all $f \in 2 B a\left(X^{*}\right)$ there exists $j^{\prime} \in$ $\left\{j_{0}, j_{0}+1, j_{0}+2, \ldots, m\right\}$ with $\left|f\left(x_{i_{0}, j^{\prime}}\right)\right|<\delta$.

Otherwise assume that for all $m \in \mathbb{N}$ there exists $x_{m}^{*} \in 2 B a\left(X^{*}\right)$ with $\left|x_{m}^{*}\left(x_{i_{0}, j}\right)\right| \geq \delta$ for $j \in\left\{j_{0}, \ldots, m\right\}$. By passing to a subsequence and relabeling assume that $\left(x_{m}^{*}\right)$ converges weak* to some $x^{*} \in 2 B a\left(X^{*}\right)$. Then $\left|x^{*}\left(x_{i_{0}, j}\right)\right| \geq \delta$ for all $j \geq j_{0}$, which contradicts that each row, in particular $\left(x_{i_{0}, j}\right)_{j=i_{0}}^{\infty}$, is weakly null.

Let $\mathrm{N}=\{m+1, m+2, \ldots\}$. Divide the set $[N]^{p}$ of all $p$-element subsets of $\mathrm{N}$ as follows: $[N]^{p}=\cup_{j=j_{0}}^{m+1} \mathcal{A}_{j}$ where for $j \in\left\{j_{0}, j_{0}+1, j_{0}+2, \ldots, m\right\}$ we set

$$
\begin{array}{r}
\mathcal{A}_{j}=\left\{F \in[N]^{p}: \text { there exists } f \in \mathcal{F} \text { having pattern } \vec{a} \text { on }\left(k_{0}, F\right)\right. \\
\text { with respect to } \left.\left(x_{i, j}\right)_{(i, j) \in I} \text { and }\left|f\left(x_{i_{0}, j}\right)\right|<\delta\right\}
\end{array}
$$

and

$\mathcal{A}_{m+1}=\left\{F \in[N]^{p}:\right.$ there is no $f \in \mathcal{F}$ having pattern $\vec{a}$ on $\left(k_{0}, F\right)$ with respect to $\left.\left(x_{i, j}\right)_{(i, j) \in I}\right\}$.

By Ramsey's theorem there exist a subsequence $\left(m_{i}\right)_{i=1}^{\infty} \in[N]$, and $j^{\prime} \in\left\{j_{0}, j_{0}+1, \ldots, m+\right.$ 1 ) such that $\left[\left(m_{i}\right)_{i=1}^{\infty}\right]^{p} \subset \mathcal{A}_{j^{\prime}}$ (where for an infinite subset $M$ of $\mathbb{N},[M]$ denotes the set of 
all infinite subsequences of $M)$. We then can pass to a subarray $\left(y_{i, j}\right)_{(i, j) \in I}$ of $\left(x_{i, j}\right)_{(i, j) \in I}$ by setting

$$
y_{i, j}= \begin{cases}x_{i, j} & \text { if }(i, j)<_{r \ell}\left(i_{0}, j_{0}\right) \\ x_{i, j^{\prime}} & \text { if }(i, j)=\left(i_{0}, j_{0}\right) \\ x_{i, m_{j}} & \text { if }\left(i_{0}, j_{0}\right)<_{r \ell}(i, j) .\end{cases}
$$

Then $\left(y_{i, j}\right)_{(i, j) \in I}$ satisfies the conclusion of the lemma, since if for some $F \subseteq\left\{k_{0}, k_{0}+1, \ldots\right\}$ with $\left(i_{0}, j_{0}\right)<_{r \ell}\left(k_{0}, \min (F)\right)$ and $|F|=p$ there exists $f \in \mathcal{F}$ having pattern $\vec{a}$ on $\left(k_{0}, F\right)$ with respect to $\left(y_{i, j}\right)_{(i, j) \in I}$, then the integer $j^{\prime}$ that was obtained by Ramsey's theorem could not be equal to $m+1$, hence $j^{\prime} \in\left\{j_{0}, j_{0}+1, \ldots, m\right\}$ and the definition of $A_{j^{\prime}}$ gives the conclusion.

Lemma 2.5. Let $\left(x_{i, j}\right)_{(i, j) \in I}$ a be regular array in a Banach space $X, \vec{A}$ be a finite set of patterns, $\mathcal{F} \subseteq 2 B a\left(X^{*}\right), \delta>0$ and $i_{0}, k_{0} \in \mathbb{N}$. Then there exists some subarray $\left(y_{i, j}\right)_{(i, j) \in I}$ of $\left(x_{i, j}\right)_{(i, j) \in I}$ such that for any $\vec{a}$ in $\vec{A}, F \subseteq\left\{k_{0}, k_{0}+1, k_{0}+2, \ldots\right\}$, with $|F|=|\vec{a}|$ and $j_{0} \in \mathbb{N}$ with $j_{0} \geq i_{0}$ and $\left(i_{0}, j_{0}\right)<_{r \ell}\left(k_{0}, \min (F)\right)$, we have the following:

If there exists $f \in \mathcal{F}$ having pattern $\vec{a}$ on $\left(k_{0}, F\right)$ with respect to $\left(y_{i, j}\right)_{(i, j) \in I}$ then there exists $g \in \mathcal{F}$ having pattern $\vec{a}$ on $\left(k_{0}, F\right)$ with respect to $\left(y_{i, j}\right)_{(i, j) \in I}$ and $\left|g\left(y_{i_{0}, j_{0}}\right)\right|<\delta$.

Additionally, we can assume that $x_{i, j}=y_{i, j}$ for all $(i, j)<_{r \ell}\left(i_{0}, i_{0}\right)$.

Proof. We begin by fixing one particular element $\vec{a}$ in $\vec{A}$. Now apply Lemma 2.4 for $\left(x_{i, j}\right)_{(i, j) \in I}, \vec{a}, \mathcal{F}, \delta, i_{0}, k_{0}$ and $j_{0}=i_{0}$ to obtain some subarray $\left(y_{i, j}^{\vec{a}, i_{0}}\right)_{(i, j) \in I}$ of $\left(x_{i, j}^{\vec{a}, i_{0}}\right)_{(i, j) \in I}$ with the property that for any $F \subseteq\left\{k_{0}, k_{0}+1, k_{0}+2, \ldots\right\}$, with $\left(i_{0}, j_{0}\right)<_{r \ell}\left(k_{0}, \min (F)\right)$ and $|F|=|\vec{a}|$ we have the following. If there exists $f \in \mathcal{F}$ having pattern $\vec{a}$ on $\left(k_{0}, F\right)$ with respect to $\left(y_{i, j}^{\vec{a}, i_{0}}\right)_{(i, j) \in I}$ then there exists $g \in \mathcal{F}$ having pattern $\vec{a}$ on $\left(k_{0}, F\right)$ with respect to $\left(y_{i, j}^{\vec{a}, i_{0}}\right)_{(i, j) \in I}$ and $\left|g\left(y_{i_{0}, j_{0}}^{\vec{a}, i_{0}}\right)\right|<\delta$. Moreover, $y_{i, j}^{\vec{a}, i_{0}}=x_{i, j}$ for all $(i, j)<_{r \ell}\left(i_{0}, j_{0}\right)$.

We repeat inductively on $j_{0}$ counting upward from $i_{0}$. Thus we next apply Lemma 2.4 to $\left(y_{i, j}^{\vec{a}, i_{0}}\right)_{(i, j) \in I}, \vec{a}, \mathcal{F}, \delta, i_{0}, k_{0}, j_{0}=i_{0}+1$, to obtain some subarray $\left(y_{i, j}^{\vec{a}, i_{0}+1}\right)_{(i, j) \in I}$ of $\left(y_{i, j}^{\vec{a}, i_{0}}\right)_{(i, j) \in I}$ with the property that for any $F \subseteq\left\{k_{0}, k_{0}+1, k_{0}+2, \ldots\right\}$ with $\left(i_{0}, j_{0}\right)<_{r \ell}\left(k_{0}, \min (F)\right)$ and $|F|=|\vec{a}|$ we have the following. If there exists $f \in \mathcal{F}$ having pattern $\vec{a}$ on $\left(k_{0}, F\right)$ with respect to $\left(y_{i, j}^{\vec{a}, i_{0}+1}\right)_{(i, j) \in I}$ then

- there exists $g \in \mathcal{F}$ having pattern $\vec{a}$ on $\left(k_{0}, F\right)$ with respect to $\left(y_{i, j}^{\vec{a}, i_{0}+1}\right)_{(i, j) \in I}$ and $\left|g\left(y_{i_{0}, i_{0}}^{\vec{a}, i_{0}+1}\right)\right|<\delta\left(\right.$ since $\left.y_{i_{0}, i_{0}}^{\vec{a}, i_{0}+1}=y_{i_{0}, i_{0}}^{\vec{a}, i_{0}}\right)$ and

- there exists $h \in \mathcal{F}$ having pattern $\vec{a}$ on $\left(k_{0}, F\right)$ with respect to $\left(y_{i, j}^{\vec{a}, i_{0}+1}\right)_{(i, j) \in I}$ and $\left|h\left(y_{i_{0}, i_{0}+1}^{\vec{a}, i_{0}+1}\right)\right|<\delta$.

Moreover, $y_{i, j}^{\vec{a}, i_{0}+1}=y_{i, j}^{\vec{a}, i_{0}}$ for all $(i, j)<_{r \ell}\left(i_{0}, i_{0}+1\right)$.

Continue in this manner for each $j_{0} \in\left\{i_{0}+2, i_{0}+3, \ldots\right\}$. Note that by fixing the elements of the subarray for $(i, j)<_{r \ell}\left(i_{0}, j_{0}\right)$ at each step $j_{0}$, there exists a subarray after infinitely many steps which possesses the properties of all the previous subarrays. We call this "limit" subarray $\left(y_{i, j}^{\vec{a}}\right)_{(i, j) \in I}$ and notice it has the property that for any $F \subseteq\left\{k_{0}, k_{0}+1, k_{0}+2, \ldots\right\}$ with $|F|=|\vec{a}|$ and for all $j_{0} \in \mathbb{N}$ with $\left(i_{0}, j_{0}\right)<_{r \ell}\left(k_{0}, \min (F)\right)$, we have the following: 
If there exists $f \in \mathcal{F}$ having pattern $\vec{a}$ on $\left(k_{0}, F\right)$ with respect to $\left(y_{i, j}^{\vec{a}}\right)_{(i, j) \in I}$ then there exists $g \in \mathcal{F}$ having pattern $\vec{a}$ on $\left(k_{0}, F\right)$ with respect to $\left(y_{i, j}^{\vec{a}}\right)_{(i, j) \in I}$ and $\left|g\left(y_{i_{0}, j_{0}}^{\vec{a}}\right)\right|<\delta$.

Notice also that any further subarray of $\left(y_{i, j}^{\vec{a}}\right)_{(i, j) \in I}$ has this same property. Then repeat the above process for each $\vec{a} \in \vec{A}$ to obtain the desired array.

Notice that if $\left(y_{i, j}\right)_{(i, j) \in I}$ is the result of applying Lemma 2.4 to some regular array and $\left(z_{i, j}\right)_{(i, j) \in I}$ is a subarray of $\left(y_{i, j}\right)_{(i, j) \in I}$ then $\left(z_{i, j}\right)_{(i, j) \in I}$ may not satisfy the conclusion of Lemma 2.4. However, if $\left(y_{i, j}\right)_{(i, j) \in I}$ is the result of applying Lemma 2.5 to some regular array and $\left(z_{i, j}\right)_{(i, j) \in I}$ is a regular subarray of $\left(y_{i, j}\right)_{(i, j) \in I}$ then $\left(z_{i, j}\right)_{(i, j) \in I}$ does satisfy the conclusion of Lemma 2.5. This idea is summarized in the following remark.

Remark 2.6. Let $\left(x_{i, j}\right)_{(i, j) \in I}$ be a regular array in a Banach space $X, \vec{A}$ be a finite set of patterns, $\mathcal{F} \subseteq 2 B a\left(X^{*}\right), i_{0}, k_{0} \in \mathbb{N}$ and $\delta>0$. Then there exists a subarray $\left(y_{i, j}\right)_{(i, j) \in I}$ of $\left(x_{i, j}\right)_{(i, j) \in I}$ such that if $\left(z_{i, j}\right)_{(i, j) \in I}$ is any subarray of $\left(y_{i, j}\right)_{(i, j) \in I}$, then for any $\vec{a}$ in $\vec{A}$, $F \subseteq\left\{k_{0}, k_{0}+1, k_{0}+2, \ldots\right\}$, with $|F|=|\vec{a}|$ and $j_{0} \in \mathbb{N}$ with $\left(i_{0}, j_{0}\right)<_{r \ell}\left(k_{0}, \min (F)\right)$, we have the following:

If there exists $f \in \mathcal{F}$ having pattern $\vec{a}$ on $\left(k_{0}, F\right)$ with respect to $\left(z_{i, j}\right)_{(i, j) \in I}$ then there exists $g \in \mathcal{F}$ having pattern $\vec{a}$ on $\left(k_{0}, F\right)$ with respect to $\left(z_{i, j}\right)_{(i, j) \in I}$ and $\left|g\left(z_{i_{0}, j_{0}}\right)\right|<\delta$.

Additionally, we can assume that $x_{i, j}=y_{i, j}$ for all $(i, j)<_{r \ell}\left(i_{0}, i_{0}\right)$.

Lemma 2.7. Let $\left(x_{i, j}\right)_{(i, j) \in I}$ be a regular array in a Banach space $X, \vec{A}$ be a finite set of patterns, $\mathcal{F} \subseteq 2 B a\left(X^{*}\right)$ and $\delta>0$. Then there exists some subarray $\left(y_{i, j}\right)_{(i, j) \in I}$ of $\left(x_{i, j}\right)_{(i, j) \in I}$ such that for all $\vec{a}$ in $\vec{A}, k_{0} \in \mathbb{N}, F \subseteq\left\{k_{0}, k_{0}+1, k_{0}+2, \ldots\right\}$ with $|F|=|\vec{a}|$ and $\left(i_{0}, j_{0}\right) \in I$ with $\left(1, k_{0}\right) \leq_{r \ell}\left(i_{0}, j_{0}\right)<_{r \ell}\left(k_{0}, \min (F)\right)$ we have the following:

If there exists $f \in \mathcal{F}$ having pattern $\vec{a}$ on $\left(k_{0}, F\right)$ with respect to $\left(y_{i, j}\right)_{(i, j) \in I}$ then there exists $g \in \mathcal{F}$ having pattern $\vec{a}$ on $\left(k_{0}, F\right)$ with respect to $\left(y_{i, j}\right)_{(i, j) \in I}$ and $\left|g\left(y_{i_{0}, j_{0}}\right)\right|<\delta$.

Proof. We will apply Remark 2.6 inductively with the subarray changing at each step, but $\vec{A}, \mathcal{F}$ and $\delta$ remaining as in the hypothesis and $\left(i_{0}, k_{0}\right)$ cycling through $\mathbb{N}^{2}$. We create the final subarray $\left(y_{i, j}\right)_{(i, j) \in I}$ of $\left(x_{i, j}\right)_{(i, j) \in I}$ inductively one column at a time. At the $j_{0}$ step of the induction we create a subarray $\left(y_{i, j}^{j_{0}}\right)_{(i, j) \in I}$ of $\left(y_{i, j+j_{0}-1}^{j_{0}-1}\right)_{(i, j) \in I}$ (where for $j_{0}=1$, $\left.\left(y_{i, j}^{0}\right)_{(i, j) \in I}=\left(x_{i, j}\right)_{(i, j) \in I}\right)$ and we set $y_{i, j_{0}}=y_{i, j_{0}}^{j_{0}}$ for $i \in\left\{1,2, \ldots, j_{0}\right\}$.

COLUMN 1: Apply Remark 2.6 to $\left(x_{i, j}\right)_{(i, j) \in I}, \vec{A}, \mathcal{F}, \delta, i_{0}=1$, and $k_{0}=1$ to obtain a subarray $\left(y_{i, j}^{1}\right)_{(i, j) \in I}$ with the property that for all $\vec{a}$ in $\vec{A}, F \subseteq\{1,2, \ldots\}$ with $|F|=|\vec{a}|$ such that $(1,1)<_{r \ell}\left(k_{0}, \min (F)\right)$ we have the following:

If there exists $f \in \mathcal{F}$ having pattern $\vec{a}$ on $\left(k_{0}, F\right)$ with respect to $\left(y_{i, j}^{1}\right)_{(i, j) \in I}$ then there exists $g \in \mathcal{F}$ having pattern $\vec{a}$ on $\left(k_{0}, F\right)$ with respect to $\left(y_{i, j}^{1}\right)_{(i, j) \in I}$ and $\left|g\left(y_{1,1}^{1}\right)\right|<\delta$.

We then fix column 1 of $\left(y_{i, j}\right)_{(i, j) \in I}$ by setting $y_{1,1}:=y_{1,1}^{1}$.

COLUMN 2: Apply Remark 2.6 to $\left(y_{i, j+1}^{1}\right)_{(i, j) \in I}, \vec{A}, \mathcal{F}, \delta$, successively for each $\left(i_{0}, k_{0}\right) \in$

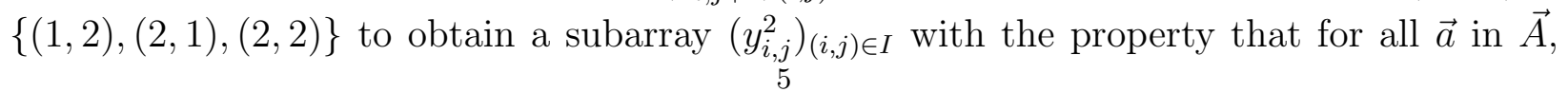


$F \subseteq\{2,3, \ldots\}$ with $|F|=|\vec{a}|, i_{0} \in\{1,2\}$ and $k_{0} \in\{1,2\}$ such that $\left(i_{0}, 2\right)<_{r \ell}\left(k_{0}, \min (F)\right)$ we have the following:

If there exists $f \in \mathcal{F}$ having pattern $\vec{a}$ on $\left(k_{0}, F\right)$ with respect to $\left(y_{i, j}^{2}\right)_{(i, j) \in I}$ then there exists $g \in \mathcal{F}$ having pattern $\vec{a}$ on $\left(k_{0}, F\right)$ with respect to $\left(y_{i, j}^{2}\right)_{(i, j) \in I}$ and $\left|g\left(y_{i_{0}, 2}^{2}\right)\right|<\delta$.

We then fix column 2 of $\left(y_{i, j}\right)_{(i, j) \in I}$ by setting $y_{i, 2}:=y_{i, 2}^{2}$ for $i \in\{1,2\}$.

COLUMN jo $\mathbf{j}_{\mathbf{0}}$ : Apply Remark 2.6 to $\left(y_{i, j+j_{0}-1}^{j_{0}-1}\right)_{(i, j) \in I}, \vec{A}, \mathcal{F}, \delta$, successively for each $\left(i_{0}, k_{0}\right) \in$ $\left\{\left(i, j_{0}\right): 1 \leq i<j_{0}\right\} \cup\left\{\left(j_{0}, j\right): 1 \leq j \leq j_{0}\right\}$ to obtain a subarray $\left(y_{i, j}^{j_{0}}\right)_{(i, j) \in I}$ with the property that for all $\vec{a}$ in $\vec{A}, F \subseteq\left\{j_{0}, j_{0}+1, \ldots\right\}$ with $|F|=|\vec{a}|, i_{0} \in\left\{1,2, \ldots, j_{0}\right\}$ and $k_{0} \in\left\{1,2, \ldots, j_{0}\right\}$ such that $\left(i_{0}, j_{0}\right)<_{r \ell}\left(k_{0}, \min (F)\right)$ we have the following:

If there exists $f \in \mathcal{F}$ having pattern $\vec{a}$ on $\left(k_{0}, F\right)$ with respect to $\left(y_{i, j}^{j_{0}}\right)_{(i, j) \in I}$ then there exists $g \in \mathcal{F}$ having pattern $\vec{a}$ on $\left(k_{0}, F\right)$ with respect to $\left(y_{i, j}^{j_{0}}\right)_{(i, j) \in I}$ and $\left|g\left(y_{i_{0}, j_{0}}^{j_{0}}\right)\right|<\delta$.

We then fix column $j_{0}$ of $\left(y_{i, j}\right)_{(i, j) \in I}$ by setting $y_{i, j_{0}}:=y_{i, j_{0}}^{j_{0}}$ for $i \in\left\{1,2, \ldots, j_{0}\right\}$.

Let $\vec{a}$ in $\vec{A}, k_{0} \in \mathbb{N}, F \subseteq\left\{k_{0}, k_{0}+1, k_{0}+2, \ldots\right\}$ with $|F|=|\vec{a}|$ and $\left(i_{0}, j_{0}\right) \in I$ with $\left(1, k_{0}\right) \leq_{r \ell}\left(i_{0}, j_{0}\right)<_{r \ell}\left(k_{0}, \min (F)\right)$ all be given. Since $\left(1, k_{0}\right) \leq_{r \ell}\left(i_{0}, j_{0}\right)$ we have that $k_{0} \leq j_{0}$. Since $\left(i_{0}, j_{0}\right)<_{r \ell}\left(k_{0}, \min (F)\right)$ we have that there exists a set $G \subseteq \mathbb{N}$ with $|G|=|F|$, $\min (G) \geq k_{0}$ and $\left(y_{k_{0}, j}\right)_{j \in F}=\left(y_{k_{0}, j}^{j_{0}}\right)_{j \in G}$. Thus if there exists $f \in \mathcal{F}$ which has pattern $\vec{a}$ on $\left(k_{0}, F\right)$ with respect to $\left(y_{i, j}\right)_{(i, j) \in I}$ then $f$ has pattern $\vec{a}$ on $\left(k_{0}, G\right)$ with respect to $\left(y_{i, j}^{j_{0}}\right)_{(i, j) \in I}$, therefore by the property of $\left(y_{i, j}^{j_{0}}\right)_{(i, j) \in I}$ we obtain that there exists $g \in \mathcal{F}$ which has pattern $\vec{a}$ on $\left(k_{0}, G\right)$ with respect to $\left(y_{i, j}^{j_{0}}\right)_{(i, j) \in I}$ and $\left|g\left(y_{i_{0}, j_{0}}^{j_{0}}\right)\right|<\delta$. Hence $g$ has pattern $\vec{a}$ on $\left(k_{0}, F\right)$ with respect to $\left(y_{i, j}\right)_{(i, j) \in I}$ and $\left|g\left(y_{i_{0}, j_{0}}\right)\right|<\delta$.

Lemma 2.8. Let $\left(x_{i, j}\right)_{(i, j) \in I}$ be a regular array in a Banach space $X, \varepsilon>0$, and $k \in \mathbb{N}$. Then there exists some subarray $\left(y_{i, j}\right)_{(i, j) \in I}$ of $\left(x_{i, j}\right)_{(i, j) \in I}$ such that for any pattern $\vec{a}$ in $[-1,1]^{p}$ for some $p \leq k$, for any $k_{0} \in \mathbb{N}$ and any $F \subseteq\left\{k_{0}, k_{0}+1, k_{0}+2, \ldots\right\}$ with $|F|=|\vec{a}|$ we have the following:

If there exists $f \in B a X^{*}$ having pattern $\vec{a}$ on $\left(k_{0}, F\right)$ with respect to $\left(y_{i, j}\right)_{(i, j) \in I}$ then there exists $g \in(1+\varepsilon) B a X^{*}$ having pattern $\vec{a}$ on $\left(k_{0}, F\right)$ with respect to $\left(y_{i, j}\right)_{(i, j) \in I}$ and

$$
\sum_{\left\{(i, j) \in I: j \geq k_{0}\right\} \backslash\left\{\left(k_{0}, \ell\right): \ell \in F\right\}}\left|g\left(y_{i, j}\right)\right|<\varepsilon
$$

Proof. Let $\left(\delta_{j}\right)_{j=0}^{\infty} \subseteq(0,1)$ such that

$$
\frac{1}{\inf _{i, j}\left\|x_{i, j}\right\|}\left(4 C k \delta_{0}+\sum_{j=1}^{\infty} 4 C j \delta_{j}\right)<\varepsilon
$$

where $C$ is the basis constant for the regular array $\left(x_{i, j}\right)_{(i, j) \in I}$. Let $A_{0}$ be a $\delta_{0}$ net for $[-1,1]$ containing zero and for each $j \in \mathbb{N}$ choose a $\delta_{j}$ net $B_{j}$ for $[-1,1]$ with $\{0\} \subseteq A_{0} \subseteq B_{1} \subseteq$ $B_{2} \subseteq \cdots$. Let

$$
\vec{A}=\left\{\vec{a}=\left(a_{i}\right)_{i=1}^{p} \in A_{0}^{p}: \text { where } 1 \leq p \leq k\right\}
$$


and

$$
\mathcal{F}=\left\{f \in\left(1+\frac{\varepsilon}{2}\right) B a\left(X^{*}\right): f\left(x_{i, j}\right) \in B_{j} \text { for all }(i, j) \in I\right\},
$$

where with out loss of generality we assume $\varepsilon<2$ so $\mathcal{F} \subseteq 2 B a\left(X^{*}\right)$. Since $0 \in A_{0}$ the zero functional is in $\mathcal{F}$ therefore $\mathcal{F}$ is nonempty .

We construct the subarray $\left(y_{i, j}\right)_{(i, j) \in I}$ of $\left(x_{i, j}\right)_{(i, j) \in I}$ inductively. First we will construct a subarray $\left(y_{i, j}^{1}\right)_{(i, j) \in I}$ of $\left(x_{i, j}\right)_{(i, j) \in I}$, then for $j \in \mathbb{N}$ for $j \geq 2$ we will construct a subarray $\left(y_{i, k}^{j}\right)_{(i, k) \in I}$ of $\left(y_{i, k+j-1}^{j-1}\right)_{(i, k) \in I}$. Once the subarray $\left(y_{i, k}^{j}\right)_{(i, k) \in I}$ has been constructed we set $y_{i, j}:=y_{i, j}^{j}$ for $1 \leq i \leq j$. Since $\left(y_{i, k}^{j}\right)_{(i, k) \in I}$ is a subarray of $\left(y_{i, k+j-1}^{j-1}\right)_{(i, k) \in I}$ and $y_{i, j}=y_{i, j}^{j}$ for $1 \leq i \leq j$, we have that $\left(y_{i, j}\right)_{(i, j) \in I}$ is a subarray of $\left(x_{i, j}\right)_{(i, j) \in I}$.

Apply Lemma 2.7 to $\left(x_{i, j}\right)_{(i, j) \in I}, \vec{A}, \delta_{1}, \mathcal{F}$ to obtain a subarray $\left(y_{i, j}^{1}\right)_{(i, j) \in I}$ of $\left(x_{i, j}\right)_{(i, j) \in I}$ such that for all $\vec{a} \in \vec{A}, k_{0} \in \mathbb{N}, F \subseteq\left\{k_{0}, k_{0}+1, k_{0}+2, \ldots\right\}$ with $|F|=|\vec{a}|$ and $\left(i_{0}, j_{0}\right) \in I$ with $\left(1, k_{0}\right) \leq_{r \ell}\left(i_{0}, j_{0}\right)<_{r \ell}\left(k_{0}, \min (F)\right)$ we have: if there exists $f \in \mathcal{F}$ having pattern $\vec{a} \in \vec{A}$ on $\left(k_{0}, F\right)$ with respect to $\left(y_{i, j}^{1}\right)_{(i, j) \in I}$ then there exists $g \in \mathcal{F}$ having pattern $\vec{a}$ on $\left(k_{0}, F\right)$ with respect to $\left(y_{i, j}^{1}\right)_{(i, j) \in I}$ and $\left|g\left(y_{i_{0}, j_{0}}^{1}\right)\right|<\delta_{1}$. Define the elements of the first column of $\left(y_{i, j}\right)_{(i, j) \in I}$ by setting $y_{1,1}:=y_{1,1}^{1}$. Define for each $b \in B_{1}$ the set

$$
\mathcal{F}_{b}=\left\{f \in \mathcal{F}: f\left(y_{1,1}\right)=b\right\} .
$$

Apply Lemma 2.7 to $\left(y_{i, j+1}^{1}\right)_{(i, j) \in I}, \vec{A}, \delta_{2}, \mathcal{F}_{b}$ successively for each $b \in B_{1}$ to obtain a subarray $\left(y_{i, j}^{2}\right)_{(i, j) \in I}$ of $\left(y_{i, j+1}^{1}\right)_{(i, j) \in I}$ such that for all $\vec{a} \in \vec{A}, k_{0} \in \mathbb{N}, F \subseteq\left\{k_{0}, k_{0}+1, k_{0}+2, \ldots\right\}$ with $|F|=|\vec{a}|$ and $\left(i_{0}, j_{0}\right) \in I$ with $\left(1, k_{0}\right) \leq_{r \ell}\left(i_{0}, j_{0}\right)<_{r \ell}\left(k_{0}, \min (F)\right)$ we have for all $b \in B_{1}$ : if there exists $f \in \mathcal{F}_{b}$ having pattern $\vec{a} \in \vec{A}$ on $\left(k_{0}, F\right)$ with respect to $\left(y_{i, j}^{2}\right)_{(i, j) \in I}$ then there exists $g \in \mathcal{F}_{b}$ having pattern $\vec{a}$ on $\left(k_{0}, F\right)$ with respect to $\left(y_{i, j}^{2}\right)_{(i, j) \in I}$ and $\left|g\left(y_{i_{0}, j_{0}}^{2}\right)\right|<\delta_{2}$.

Define the elements of the second column of $\left(y_{i, j}\right)_{(i, j) \in I}$ by setting $y_{1,2}:=y_{1,2}^{2}$, and $y_{2,2}:=$ $y_{2,2}^{2}$. For each $\vec{b}=\left(b_{1}, b_{2}, b_{3}\right) \in B_{1} \times B_{2} \times B_{2}$ set

$$
\mathcal{F}_{\vec{b}}=\left\{f \in \mathcal{F}: f\left(y_{1,1}\right)=b_{1}, f\left(y_{1,2}\right)=b_{2} \text { and } f\left(y_{2,2}\right)=b_{3}\right\} .
$$

Apply Lemma 2.7 to $\left(y_{i, j+2}^{2}\right)_{(i, j) \in I}, \vec{A}, \delta_{3}, \mathcal{F}_{\vec{b}}$ successively for each $\vec{b} \in B_{1} \times B_{2} \times B_{2}$ to obtain a subarray $\left(y_{i, j}^{3}\right)_{(i, j) \in I}$ of $\left(y_{i, j+2}^{2}\right)_{(i, j) \in I}$ such that for all $\vec{a} \in \vec{A}, k_{0} \in \mathbb{N}, F \subseteq$ $\left\{k_{0}, k_{0}+1, k_{0}+2, \ldots\right\}$ with $|F|=|\vec{a}|$ and $\left(i_{0}, j_{0}\right) \in I$ with $\left(1, k_{0}\right) \leq_{r \ell}\left(i_{0}, j_{0}\right)<_{r \ell}\left(k_{0}, \min (F)\right)$ we have for all $\vec{b} \in B_{p_{1,1}} \times B_{p_{1,2}} \times B_{p_{2,2}}$ : if there exists $f \in \mathcal{F}_{\vec{b}}$ having pattern $\vec{a} \in \vec{A}$ on $\left(k_{0}, F\right)$ with respect to $\left(y_{i, j}^{3}\right)_{(i, j) \in I}$ then there exists $g \in \mathcal{F}_{\vec{b}}$ having pattern $\vec{a}$ on $\left(k_{0}, F\right)$ with respect to $\left(y_{i, j}^{3}\right)_{(i, j) \in I}$ and $\left|g\left(y_{i_{0}, j_{0}}^{3}\right)\right|<\delta_{3}$. Define the elements in the third column $\left(y_{i, j}\right)_{(i, j) \in I}$ by setting $y_{1,3}:=y_{1,3}^{3}, y_{2,3}:=y_{2,3}^{3}$ and $y_{3,3}:=y_{2,3}^{3}$. Continue in this manner to create the subarray $\left(y_{i, j}\right)_{(i, j) \in I}$ of $\left(x_{i, j}\right)_{(i, j) \in I}$.

Let $\tilde{f} \in B a\left(X^{*}\right), \vec{c}$ be a $p$-pattern for $p \leq k, k_{0} \in \mathbb{N}$ and $F \subseteq\left\{k_{0}, k_{0}+1, k_{0}+2, \ldots\right\}$ with $|F|=p$ such that $\tilde{f}$ has pattern $\vec{c}$ on $\left(k_{0}, F\right)$ with respect to $\left(y_{i, j}\right)_{(i, j) \in I}$.

First it is easy to see using (3) that since $\tilde{f} \in B a\left(X^{*}\right)$ there is $f \in \mathcal{F}$ (as defined in (5)) such that

- for all $j \in F$ we have $f\left(y_{k_{0}, j}\right) \in A_{0}$ and $\left|\tilde{f}\left(y_{k_{0}, j}\right)-f\left(y_{k_{0}, j}\right)\right| \leq \delta_{0}$, and

- if we define the finite set $G \subset \mathbb{N}$ by $\left(y_{k_{0}, j}\right)_{j \in F}=\left(x_{k_{0}, j}\right)_{j \in G}$, then for all $(i, j) \in$ $I \backslash\left\{\left(k_{0}, j\right): j \in G\right\}$ we have that $\left|\tilde{f}\left(x_{i, j}\right)-f\left(x_{i, j}\right)\right| \leq \delta_{j}$ and $f\left(x_{i, j}\right) \in B_{j}$. 
Let $\vec{a}:=\left(f\left(y_{k_{0}, j}\right)\right)_{j \in F}$ and note that $f$ has pattern $\vec{a}$ on $\left(k_{0}, F\right)$ with respect to $\left(y_{i, j}\right)_{(i, j) \in I}$. We will find a functional $g \in\left(1+\frac{\varepsilon}{2}\right) B a\left(X^{*}\right)$ such that $g$ has pattern $\vec{a}$ on $F$ with respect to $\left(y_{i, j}\right)_{(i, j) \in I}$ and $\sum_{I^{\prime} \backslash\left\{\left(k_{0}, j\right): j \in F\right\}}\left|g\left(y_{i, j}\right)\right|<\varepsilon$.

We proceed to find such functional $g$. But first a bit of notation, for a $p$-pattern $\vec{\alpha}=\left(\alpha_{i}\right)_{i=1}^{p}$ we define its derivative $\vec{\alpha}^{\prime}=\left(\alpha_{i-1}\right)_{i=2}^{p}$.

We will walk through the index set $I^{\prime}=\left\{(i, j) \in I: j \geq k_{0}\right\}$ proceeding through this set in $<_{r \ell}$-order and at each step find a functional $g_{i, j}$ with the property that if $(i, j) \notin$ $\left\{\left(k_{0}, j\right): j \in F\right\}$ then $\left|g_{i, j}\left(y_{i, j}\right)\right|$ will be small and "agree" with the previous functional on $\left\{\left(i^{\prime}, j^{\prime}\right) \in I^{\prime}:\left(i^{\prime}, j^{\prime}\right)<_{r \ell}(i, j)\right\}$. If $(i, j) \in\left\{\left(k_{0}, j\right): j \in F\right\}$ then we will not change the previously defined functional. We will assume $k_{0} \geq 3$ for purposes of demonstrating the construction, but if $k_{0}=1$ or 2 then we proceed similarly.

$\operatorname{STEP}\left(1, k_{0}\right)$ : Note $\left(1, k_{0}\right) \notin\left\{\left(k_{0}, j\right): j \in F\right\}$ (since $\left.k_{0} \geq 3\right)$. Let

$$
\vec{b}=\left(f\left(y_{1,1}\right), f\left(y_{2,1}\right), f\left(y_{2,2}\right), f\left(y_{3,1}\right), \ldots,, f\left(y_{k_{0}-1, k_{0}-1}\right)\right) .
$$

Then $f \in \mathcal{F}_{\vec{b}}, f$ has pattern $\vec{a}$ on $\left(k_{0}, F\right)$ with respect to $\left(y_{i, j}\right)_{(i, j) \in I},\left(y_{i, j}\right)_{(i, j) \in I^{\prime}}$ is a subarray of $\left(y_{(i, j)}^{k_{0}}\right)_{(i, j) \in I^{\prime}}$ and $\left(1, k_{0}\right) \leq_{r \ell}\left(1, k_{0}\right)<_{r \ell}\left(k_{0}, \min (F)\right)$, (the last inequality is valid since $\left.k_{0} \geq 3\right)$. Thus there exists $g_{1, k_{0}} \in \mathcal{F}_{\vec{b}}$ such that $g_{1, k_{0}}$ has pattern $\vec{a}$ on $\left(k_{0}, F\right)$ with respect to $\left(y_{i, j}\right)_{(i, j) \in I}$ and $\left|g_{1, k_{0}}\left(y_{1, k_{0}}\right)\right|<\delta_{k_{0}}$. Set $F_{1, k_{0}}=F$ and $\vec{a}_{1, k_{0}}=\vec{a}$.

$\operatorname{STEP}\left(2, k_{0}\right)$ : Note that $\left(2, k_{0}\right) \notin\left\{\left(k_{0}, j\right): j \in F_{1, k_{0}}\right\}$ (since $k_{0} \geq 3$ ). Let

$$
\vec{b}=\left(f\left(y_{1,1}\right), f\left(y_{2,1}\right), f\left(y_{2,2}\right), \ldots, f\left(y_{k_{0}-1, k_{0}-1}\right), g_{1, k_{0}}\left(y_{1, k_{0}}\right)\right) .
$$

Then $g_{1, k_{0}} \in \mathcal{F}_{\text {vecb }}, g_{1, k_{0}}$ has pattern $\vec{a}_{1, k_{0}}$ on $\left.\left(k_{0}, F_{1, k_{0}}\right)\right)$ with respect to $\left(y_{i, j}\right)_{(i, j) \in I}$ and $\left(1, k_{0}\right)<_{r \ell}\left(2, k_{0}\right)<_{r \ell}\left(k_{0}, \min (F)\right)$, (the last inequality is valid because $\left.k_{0} \geq 3\right)$. Thus there exists $g_{2, k_{0}} \in \mathcal{F}_{\vec{b}}$ such that $g_{2, k_{0}}$ has pattern $\vec{a}_{1, k_{0}}$ on $\left(k_{0}, F_{1, k_{0}}\right)$ with respect to $\left(y_{i, j}\right)_{(i, j) \in I}$ and $\left|g_{2, k_{0}}\left(y_{2, k_{0}}\right)\right|<\delta_{k_{0}}$. Set $F_{2, k_{0}}=F_{1, k_{0}}$ and $\vec{a}_{2, k_{0}}=\vec{a}_{1, k_{0}}$.

We continue similarly until the $\left(k_{0}-1, k_{0}\right)$ step. The step $\left(k_{0}, k_{0}\right)$ is slightly different. We separate this step into two different cases depending on whether or not $\left(k_{0}, k_{0}\right) \in\left\{\left(k_{0}, j\right)\right.$ : $\left.j \in F_{k_{0}-1, k_{0}}\right\}$.

$\operatorname{STEP}\left(k_{0}, k_{0}\right):$ If $\left(k_{0}, k_{0}\right) \in\left\{\left(k_{0}, j\right): j \in F_{k_{0}-1, k_{0}}\right\}$ then set $g_{k_{0}, k_{0}}=g_{k_{0}-1, k_{0}}, F_{k_{0}, k_{0}}=$ $F_{k_{0}-1, k_{0}} \backslash\left\{k_{0}\right\}$ and $\vec{a}_{k_{0}, k_{0}}=\vec{a}_{k_{0}-1, k_{0}}^{\prime}$.

If $\left(k_{0}, k_{0}\right) \notin\left\{\left(k_{0}, j\right): j \in F_{k_{0}-1, k_{0}}\right\}$ then $\left(k_{0}, k_{0}\right)<_{r \ell}\left(k_{0}, \min (F)\right)$. Let

$$
\vec{b}=\left(f\left(y_{1,1}\right), f\left(y_{2,1}\right), f\left(y_{2,2}\right), \ldots, f\left(y_{k_{0}-1, k_{0}-1}\right), g_{1, k_{0}}\left(y_{1, k_{0}}\right), g_{2, k_{0}}\left(y_{2, k_{0}}\right), \ldots, g_{k_{0}-1, k_{0}}\left(y_{k_{0}-1, k_{0}}\right)\right) \text {. }
$$

Then $g_{k_{0}-1, k_{0}} \in \mathcal{F}_{\vec{b}}, g_{k_{0}-1, k_{0}}$ has pattern $\vec{a}_{k_{0}-1, k_{0}}$ on $\left(k_{0}, F_{k_{0}-1, k_{0}}\right)$ with respect to $\left(y_{i, j}\right)_{(i, j) \in I}$, $\left(y_{i, j}\right)_{(i, j) \in I^{\prime}}$ is a subarray of $\left(y_{i, j}^{k_{0}}\right)_{(i, j) \in I}$ and $\left(1, k_{0}\right) \leq_{r \ell}\left(k_{0}, k_{0}\right)<_{r \ell}\left(k_{0}, \min (F)\right)$. Thus there exists $g_{k_{0}, k_{0}} \in \mathcal{F}_{\vec{b}}$ such that $g_{k_{0}, k_{0}}$ has pattern $\vec{a}_{k_{0}-1, k_{0}}$ on $\left(k_{0}, F_{k_{0}-1, k_{0}}\right)$ with respect to $\left(y_{i, j}\right)_{(I, j) \in I}$ and $g_{k_{0}, k_{0}}\left(y_{k_{0}, k_{0}}\right)<\delta_{k_{0}}$. In this case set $F_{k_{0}, k_{0}}=F_{k_{0}-1, k_{0}}$ and $\vec{a}_{k_{0}, k_{0}}=\vec{a}_{k_{0}-1, k_{0}}$.

Then start again with the first entry $\left(1, k_{0}+1\right)$ of the next column as in steps $\left(1, k_{0}\right)$ and $\left(2, k_{0}\right)$.

$\operatorname{STEP}\left(1, k_{0}+1\right)$ : Note that $\left(1, k_{0}+1\right) \notin\left\{\left(k_{0}, j\right): j \in F_{k_{0}, k_{0}}\right\}$, (since $k_{0} \geq 3$ ). Let

$$
\vec{b}=\left(f\left(y_{1,1}\right), f\left(y_{2,1}\right), f\left(y_{2,2}\right), \ldots, g_{1, k_{0}}\left(y_{1, k_{0}}\right), g_{2, k_{0}}\left(y_{2, k_{0}}\right), g_{k_{0}, k_{0}}\left(y_{k_{0}, k_{0}}\right)\right) .
$$

Then $g_{k_{0}, k_{0}} \in \mathcal{F}_{\vec{b}}, g_{k_{0}, k_{0}}$ has pattern $\vec{a}_{k_{0}, k_{0}}$ on $\left(k_{0}, F_{k_{0}, k_{0}}\right)$ with respect to $\left(y_{i, j}\right)_{(i, j) \in I},\left(y_{i, j}\right)_{(i, j) \in I^{\prime}}$ is a subarray of $\left(y_{i, j}^{k_{0}}\right)_{(i, j) \in I}$ and $\left(1, k_{0}\right) \leq_{r \ell}\left(1, k_{0}+1\right)<_{r \ell}\left(k_{0}, \min (F)\right)$, (the last inequality is valid since $\left.k_{0} \geq 3\right)$. Thus there exists $g_{1, k_{0}+1} \in \mathcal{F}_{\vec{b}}$ such that $g_{1, k_{0}+1}$ has pattern $\vec{a}_{k_{0}, k_{0}}$ on 
$\left(k_{0}, F_{k_{0}, k_{0}}\right)$ with respect to $\left(y_{i, j}\right)_{(i, j) \in I}$ and $\left|g_{1, k_{0}+1}\left(y_{1, k_{0}+1}\right)\right|<\delta_{k_{0}+1}$. Set $F_{1, k_{0}+1}=F_{k_{0}, k_{0}}$ and $\vec{a}_{1, k_{0}+1}=\vec{a}_{k_{0}, k_{0}}$.

Continue in this manner to generate a sequence of functionals $\left(g_{i, j}\right)_{(i, j) \in I^{\prime}}$. We only need to distinguish two cases every time we reach the $k_{0}$ row as in step $\left(k_{0}, k_{0}\right)$. Let $g \in(1+$ $\left.\frac{\varepsilon}{2}\right) B a\left(X^{*}\right)$ be a weak*-accumulation point of sequence $\left(g_{i, j}\right)_{(i, j) \in I^{\prime}}$. Note $g$ has the following two properties:

- $g$ has pattern $\vec{a}$ on $\left(k_{0}, F\right)$ with respect to $\left(y_{i, j}\right)_{(i, j) \in I}$, and

- $\sum_{(i, j) \in I^{\prime} \backslash\left\{\left(k_{0}, \ell\right): \ell \in F\right\}}\left|g\left(y_{i, j}\right)\right|<\varepsilon$.

Since $g\left(y_{k_{0}, \ell}\right)=f\left(y_{k_{0}, \ell}\right) \in A_{0}$ for all $\ell \in F$ and $\left|f\left(y_{k_{0}, \ell}\right)-\tilde{f}\left(y_{k_{0}, \ell}\right)\right|<\delta_{0}$ for all $\ell \in F$, (3) implies that there exists $\tilde{g} \in X^{*}$ such that $\|\tilde{g}\| \leq\|g\|+\frac{\varepsilon}{2} \leq 1+\varepsilon$ and $\tilde{g}\left(y_{k_{0}, \ell}\right)=\tilde{f}\left(y_{k_{0}, \ell}\right) \in A_{0}$ for all $\ell \in F$ (thus $\tilde{g}$ has pattern $\vec{c}$ on $\left(k_{0}, F\right)$ with respect to $\left.\left(y_{i, j}\right)_{(i, j) \in I}\right)$ and

completing the proof.

$$
\sum_{(i, j) \in I^{\prime} \backslash\left\{\left(k_{0}, \ell\right): \ell \in F\right\}}\left|\tilde{g}\left(y_{i, j}\right)\right|<\varepsilon
$$

Finally we arrive to the

Proof of Theorem 1.2. First, by Remark 2.3 assume without loss of generality that $\left(x_{i, j}\right)_{(i, j) \in I}$ is regular. Let $\eta>0$ such that

$$
2(2 \eta+1) \leq 2+\frac{\varepsilon}{2}
$$

where $C$ is the basis constant of the regular array $\left(x_{i, j}\right)_{(i, j) \in I}$. Apply Lemma 2.8 to $\left(x_{i, j}\right)_{(i, j) \in I}$, $\eta$ and $M_{1}$ to get $\left(y_{i, j}^{1}\right)_{(i, j) \in I}$. Define $y_{1,1}:=y_{1,1}^{1}$.

Apply Lemma 2.8 to $\left(y_{i, j+1}^{1}\right)_{(i, j) \in I}, \eta$ and $M_{2}$ to get $\left(y_{i, j}^{2}\right)_{(i, j) \in I}$. Define $y_{i, 2}:=y_{i, 2}^{2}$ for $i=1,2$.

Assuming that $\left(y_{i, j}^{\ell-1}\right)_{(i, j) \in I}$ has been defined (and thus $\left(y_{i, j}\right)_{(i, j) \in I ; j<\ell}$ ) has also been defined) apply Lemma 2.8 to $\left(y_{i, j+\ell-1}^{\ell-1}\right)_{(i, j) \in I}, \eta$ and $M_{\ell}$ to get $\left(y_{i, j}^{\ell}\right)_{(i, j) \in I}$. Define $y_{i, \ell}:=y_{i, \ell}^{\ell}$ for $i=1,2, \ldots, \ell$.

Inductively construct the entire array $\left(y_{i, j}\right)_{(i, j) \in I}$ and notice $\left(y_{i, j}\right)_{(i, j) \in I}$ is regular by Remark 2.1.

Let $k_{0} \in \mathbb{N}, F \subseteq\left\{k_{0}, k_{0}+1, k_{0}+2, \ldots\right\}$ with $|F| \leq M_{\min (F)}$ and finitely supported scalars $\left(a_{i, j}\right)_{(i, j) \in I}$ be given. We can assume without loss of generality that

$$
\left\|\sum_{(i, j) \in I} a_{i, j} y_{i, j}\right\|=1 .
$$

Then $\left|a_{i, j}\right| \leq 2 C$ for $(i, j) \in I$. Let $f \in B a\left(X^{*}\right)$ such that

$$
f\left(\sum_{j \in F} a_{k_{0}, j} y_{k_{0}, j}\right)=\left\|\sum_{j \in F} a_{k_{0}, j} y_{k_{0}, j}\right\| .
$$

Let $\vec{a}=\left(f\left(y_{k_{0}, j}\right)\right)_{j \in F}$ be a $p$-pattern where $p=|F|$. Obviously $f$ has pattern $\vec{a}$ on $\left(k_{0}, F\right)$ with respect to $\left(y_{i, j}\right)_{(i, j) \in I}$. Then by considering the subarray $\left(y_{i, j}\right)_{(i, j) \in I, j \geq \min (F)}$ of $\left(y_{i, j}^{\min (F)}\right)_{(i, j) \in I: j \geq \min (F)}$ we obtain by the above that there exists $g \in(1+\eta) B a\left(X^{*}\right)$ having pattern $\vec{a}$ on $\left(k_{0}, F\right)$ with respect to $\left(y_{i, j}\right)_{(i, j) \in I}$ and $\sum_{\{(i, j) \in I: j \geq \min (F)\} \backslash\left\{\left(k_{0}, \ell\right): \ell \in F\right\}}\left|g\left(y_{i, j}\right)\right|<\eta$. Thus 


$$
\begin{aligned}
1 & =\left\|\sum a_{i, j} y_{i, j}\right\| \geq \frac{1}{2 C}\left\|\sum_{j \geq \min (F)} a_{i, j} y_{i, j}\right\| \geq \frac{1}{2 C(1+\eta)}\left|\sum_{j \geq \min (F)} a_{i, j} g\left(y_{i, j}\right)\right| \\
& \geq \frac{1}{2 C(1+\eta)}\left|\sum_{j \in F} a_{k_{0}, j} g\left(y_{k_{0}, j}\right)\right|-\frac{1}{2 C(1+\eta)}\left|\sum_{\{(i, j) \in I: j \geq \min (F)\} \backslash\left\{\left(k_{0}, j\right): j \in F\right\}} a_{i, j} g\left(y_{i, j}\right)\right| \\
& \geq \frac{1}{2 C(1+\eta)}\left\|\sum_{j \in F} a_{k_{0}, j} y_{k_{0}, j}\right\|-\frac{1}{2 C(1+\eta)}\left|a_{i, j}\right|\left|g\left(y_{i, j}\right)\right| \\
& \geq \frac{1}{2 C(1+\eta)}\left\|\sum_{j \in F} a_{k_{0}, j} y_{k_{0}, j}\right\|-\frac{1}{1+\eta} \sum_{\{(i, j): j \geq \min (F)\} \backslash\left\{\left(k_{0}, j\right): j \in F\right\}}\left|g\left(y_{i, j}\right)\right| \\
& \geq \frac{1}{2 C(1+\eta)}\left\|\sum_{j \in F} a_{k_{0}, j} y_{k_{0}, j}\right\|-\frac{1}{1+\eta} \eta .
\end{aligned}
$$

Thus by (8) and (9) we have

$$
\left\|\sum_{(i, j) \in I} a_{i, j} y_{i, j}\right\|=1 \geq \frac{1}{2 C(2 \eta+1)}\left\|\sum_{j \in F} a_{k_{0}, j} y_{k_{0}, j}\right\| \geq \frac{1}{C\left(2+\frac{\varepsilon}{2}\right)}\left\|\sum_{j \in F} a_{k_{0}, j} y_{k_{0}, j}\right\|
$$

Since we can choose $C$, the basis constant of our regular array, arbitrarily close to 1 (see Remark 2.3) we have shown the result.

\section{Existence of Non-trivial Operators}

In this section we give an application of Theorem 1.2 to the "multiple of the inclusion plus compact problem". The problem has been previously studied in [9], [2] and [21]. The main results of this section are Theorems 3.2 and 3.8. In order to formulate these results we need some definitions.

Recall that $[4,5,6]$ for every seminormalized basic sequence $\left(y_{n}\right)$ in a Banach space $X$ and for every $\left(\varepsilon_{n}\right) \searrow 0$ there exists a subsequence $\left(x_{n}\right)$ of $\left(y_{n}\right)$ and a seminormalized basic sequence $\left(\tilde{x}_{n}\right)$ (not necessarily in $X$ ) such that for all $n \in \mathbb{N}$, scalars $\left(a_{i}\right)_{i=1}^{n}$ with $\left|a_{i}\right| \leq 1$ and $n \leq k_{1}<\cdots<k_{n}$,

$$
\left|\left\|\sum_{i=1}^{n} a_{k_{i}} x_{k_{i}}\right\|-\left\|\sum_{i=1}^{n} a_{i} \tilde{x}_{i}\right\|\right|<\varepsilon_{n} .
$$

The sequence $\left(\tilde{x}_{n}\right)$ is called a spreading model of $\left(x_{n}\right)$. If $\left(x_{n}\right)$ is a seminormalized weakly null basic sequence then $\left(\tilde{x}_{n}\right)$ is an unconditional basic sequence. Thus if $X$ is an HI Banach space and $\left(x_{n}\right)$ is a seminormalized basic sequence in $X$ with spreading model $\left(\tilde{x}_{n}\right)$, then it may be easier to study $\left(\tilde{x}_{n}\right)$ than to study $\left(x_{n}\right)$ itself.

A similar notion to the next one was introduced in [21].

Definition 3.1. Let $\left(x_{n}\right)$ and $\left(z_{n}\right)$ be two seminormalized basic sequences (not necessarily in the same Banach space), such that $\left(z_{n}\right)$ is not equivalent to the unit vector basis of $c_{0}$. We 
say that $\left(z_{n}\right)$ dominates $\left(x_{n}\right)$ on small coefficients, (denoted by $\left(x_{n}\right)<<\left(z_{n}\right)$ and abbreviated as " $\left(z_{n}\right)$ s.c. dominates $\left.\left(x_{n}\right)\right)$ ", if

$$
\liminf _{\varepsilon \searrow 0}\left\{\left\|\sum a_{i} z_{i}\right\|:\left|a_{i}\right| \leq \varepsilon \text { and }\left\|\sum a_{i} x_{i}\right\|=1\right\}=\infty
$$

where $\inf \emptyset=\infty$.

Theorem 3.2. Let $X$ be a Banach space containing seminormalized basic sequences $\left(x_{i}\right)_{i}$ and $\left(x_{i}^{n}\right)_{i}$ for all $n \in \mathbb{N}$, such that $0<\inf _{n, i}\left\|x_{i}^{n}\right\| \leq \sup _{n, i}\left\|x_{i}^{n}\right\|<\infty$. Let $\left(z_{i}\right)_{i}$ be a basic sequence not necessarily in $X$. Assume that $\left(x_{i}\right)$ satisfies:

The sequence $\left(x_{i}\right)_{i}$ has a spreading model $\left(\tilde{x}_{i}\right)_{i=1}^{\infty}$ such that $\left(\tilde{x}_{i}\right)_{i \in \mathbb{N}}<<\left(z_{i}\right)_{i \in \mathbb{N}}$.

Assume that for all $n \in \mathbb{N}$ the sequence $\left(x_{i}^{n}\right)_{i}$ satisfies:

The sequence $\left(x_{i}^{n}\right)_{i}$ has a spreading model $\left(\tilde{x}_{i}^{n}\right)_{i}$ such that

$\left(z_{i}\right)_{i=1}^{n}$ is $C$-dominated by $\left(\tilde{x}_{i}^{n}\right)_{i=1}^{n}$ for some $C$ independent of $n$.

Then there exists a subspace $Y$ of $X$ which has a basis and an operator $T \in \mathcal{L}(Y, X)$ which is not a compact perturbation of a multiple of the inclusion map.

For the proof of Theorem 3.2 we need the following three lemmas whose proofs are postponed. For their formulation we need to introduce the following

Definition 3.3. A seminormalized basic sequence $\left(x_{n}\right)$ has Property $P$ if for all $\rho>0$ there exists an $M=M(\rho) \in \mathbb{N}$, such that if $\left\|\sum a_{i} z_{i}\right\|=1$ then $\left|\left\{i:\left|a_{i}\right| \geq \rho\right\}\right| \leq M$.

Obviously Property $\mathrm{P}$ can be equivalently stated with the equality " $\left\|\sum a_{i} z_{i}\right\|=1$ " replaced by " $\left\|\sum a_{i} z_{i}\right\| \leq 1$ " (and everything else staying unchanged).

Lemma 3.4. Let $X$ be a Banach space which does not contain an isomorphic copy of $\ell_{1}$ and let $\left(z_{i}\right)$ be a basic sequence not necessarily in $X$. Assume that $X$ contains basic sequences $\left(x_{i}\right)$ and $\left(x_{i}^{n}\right)$ for all $n \in \mathbb{N}$ such that $0<\inf _{n, i}\left\|x_{i}^{n}\right\| \leq \sup _{n, i}\left\|x_{i}^{n}\right\|<\infty$ and conditions (11) and (12) of Theorem 3.2 are satisfied. Then there exist seminormalized basic sequences $\left(X_{i}\right)_{i}$ and $\left(Z_{i}\right)$ not necessarily in $X$ and for each $n \in \mathbb{N}$ there exists a basic sequence $\left(X_{i}^{n}\right)_{i}$ in $X$ such that $0<\inf _{n, i}\left\|X_{i}^{n}\right\| \leq \sup _{n, i}\left\|X_{i}^{n}\right\|<\infty$ and the following conditions are satisfied:

(a) The sequence $\left(X_{i}\right)_{i}$ dominates $\left(\underline{x}_{i}\right)_{i}$, where $\left(\underline{x}_{i}\right)_{i}=\left(x_{m_{2 i}}-x_{m_{2 i-1}}\right)$ for some increasing subsequence $\left(m_{i}\right)$ of positive integers, and $\left(\underline{x}_{i}\right)$ is weakly null.

(b) $\left(X_{i}\right)$ and $\left(Z_{i}\right)$ satisfy (11) of Theorem 3.2.

(c) $\left(X_{i}\right)$ has Property $P$.

(d) $\left(X_{i}^{n}\right)_{i}$ and $\left(Z_{i}\right)$ satisfy (12) of Theorem 3.2.

(e) The sequence $\left(X_{i}^{n}\right)_{i}$ is weakly null for all $n \in \mathbb{N}$.

Lemma 3.5. Let $X$ be a Banach space, $\left(X_{i}\right)$ be a seminormalized basic sequence in $X$ having Property $P$ and $\left(Z_{i}\right)$ be a seminormalized basic sequence not necessarily in $X$. Assume that the sequence $\left(X_{i}\right)$ and $\left(Z_{i}\right)$ satisfy condition (11) of Theorem 3.2. Then for all $\left(\delta_{n}\right)_{n=2}^{\infty} \subseteq(0, \infty)$ there exists an increasing sequence $M_{1}<M_{2}<\cdots$ of positive integers and a subsequence $\left(X_{n_{i}}\right)$ of $\left(X_{i}\right)$ such that for all $\left(a_{i}\right) \in c_{00}$,

$$
\left\|\sum a_{i} X_{n_{i}}\right\| \leq \sup _{n \in \mathbb{N}} \sup _{n \leq F \subseteq \mathbb{N} ;|F| \leq M_{n}} \delta_{n}\left\|\sum_{i \in F} a_{i} Z_{i}\right\|,
$$

for some $\delta_{1}$ (where " $n \leq F$ " means $n \leq \min (F)$ ). 
Lemma 3.6. Let $X$ be a Banach space, $\left(\delta_{n}\right)$ be a summable sequence of positive numbers, $\left(M_{n}\right)_{n=1}^{\infty} \subseteq \mathbb{N}$ be a sequence of positive integers, $\left(Z_{n}\right)$ be a seminormalized basic sequence (not necessarily in $X$ ) and for every $n \in \mathbb{N}$ let $\left(X_{j}^{n}\right)_{j=1}^{\infty}$ be a weakly null basic sequence in $X$ having spreading model $\left(\tilde{X}_{j}^{n}\right)_{j=1}^{\infty}$ such that $0<\inf _{n, j}\left\|X_{j}^{n}\right\| \leq \sup _{n, j}\left\|X_{j}^{n}\right\|<\infty$ and condition (12) of Theorem 3.2 is satisfied. Then there exists a seminormalized weakly null basic sequence $\left(y_{i}\right)$ in $X$ such that

$$
\frac{1}{6 C} \sup _{n} \sup _{n \leq F \subseteq \mathbb{N} ;|F| \leq M_{n}} \delta_{n}\left\|\sum_{i \in F} a_{i} Z_{i}\right\| \leq\left\|\sum a_{i} y_{i}\right\| .
$$

Moreover, $\left\|y_{j}\right\| \geq \frac{\delta_{1}}{2} \inf _{n, m}\left\|X_{m}^{n}\right\|$. Furthermore, if $\left(x_{i}^{*}\right)$ is any given sequence of functionals in $X^{*}$ and $\varepsilon>0$ we can choose $\left(y_{i}\right)$ to satisfy $\left|x_{i}^{*} y_{i}\right|<\varepsilon$.

We now present the

Proof of Theorem 3.2. Obviously, if $\ell_{1}$ embeds in $X$, (or if, more generally, $X$ contains an unconditional basic sequence), then the conclusion of Theorem 3.2 is satisfied. Thus we can assume that $X$ does not contain an isomorphic copy of $\ell_{1}$ and consider the sequences $\left(X_{i}\right)$, $\left(Z_{i}\right),\left(X_{i}^{n}\right)$ and $\left(\underline{x}_{i}\right)$ which satisfy the conclusion of Lemma 3.4. Let $\left(\delta_{n}\right)_{n=2}^{\infty}$ be a summable sequence of positive numbers. Apply Lemma 3.5 to obtain a subsequences $\left(X_{n_{i}}\right), \delta_{1}>0$ and an increasing sequence $\left(M_{n}\right)_{n \in \mathbb{N}}$ of positive integers which satisfies (13). For every $i \in \mathbb{N}$ let a norm 1 functional $x_{i}^{*}$ satisfying $x_{i}^{*} \underline{x}_{n_{i}}=\left\|\underline{x}_{n_{i}}\right\|$. Then apply Lemma 3.6 for $\left(\delta_{n}\right)_{n \in \mathbb{N}}$ and $\left(M_{n}\right)_{n \in \mathbb{N}}$ to obtain a basic sequence $\left(y_{i}\right)$ which satisfies $(14)$.

Assume also that $\left(y_{i}\right)$ satisfies the "furthermore" part of the statement of Lemma 3.6 for the sequence $\left(x_{i}^{*}\right)$ and $\varepsilon=\delta_{1} \inf _{n, i}\left\|X_{i}^{n}\right\| \inf _{i}\left\|\underline{x}_{i}\right\| /\left(8 \sup _{i}\left\|\underline{x}_{i}\right\|\right)$. Note that if $|\lambda| \geq \frac{4 \sup _{i}\left\|\underline{x}_{i}\right\|}{\delta_{1} \inf _{n, i}\left\|X_{i}^{n}\right\|}$ then

$$
\left\|\underline{x}_{n_{j}}+\lambda y_{j}\right\| \geq|\lambda|\left\|y_{j}\right\|-\left\|\underline{x}_{n_{j}}\right\| \geq \frac{4 \sup _{i}\left\|\underline{x}_{i}\right\|}{\delta_{1} \inf _{n, i}\left\|X_{i}^{n}\right\|} \frac{\delta_{1}}{2} \inf _{n, i}\left\|X_{i}^{n}\right\|-\left\|\underline{x}_{n_{j}}\right\| \geq\left\|\underline{x}_{n_{j}}\right\| \geq \inf _{i}\left\|\underline{x}_{i}\right\|
$$

(by the "moreover" part of the statement of Lemma 3.6). Also if $|\lambda|<\frac{4 \sup _{i}\left\|x_{i}\right\|}{\delta_{1} \inf _{n, i}\left\|X_{i}^{n}\right\|}$ then

$$
\left\|\underline{x}_{n_{j}}+\lambda y_{j}\right\| \geq\left|x_{j}^{*}\left(\underline{x}_{n_{j}}+\lambda y_{j}\right)\right| \geq\left\|\underline{x}_{n_{j}}\right\|-\frac{4 \sup _{i}\left\|\underline{x}_{i}\right\|}{\delta_{1} \inf _{n, i}\left\|X_{i}^{n}\right\|} \varepsilon \geq \frac{1}{2} \inf _{i}\left\|\underline{x}_{i}\right\| .
$$

Thus for all scalars $\lambda$ we have

$$
\left\|\underline{x}_{n_{j}}+\lambda y_{j}\right\| \geq \frac{1}{2} \inf _{i}\left\|\underline{x}_{i}\right\| .
$$

Thus if we define $T:\left[\left(y_{i}\right)\right] \rightarrow X$ by

$$
T\left(\sum a_{i} y_{i}\right)=\sum a_{i} \underline{x}_{n_{i}}
$$

we have that this operator is bounded by (13), (14) and our assumption that $\left(\underline{x}_{i}\right)_{i}$ is dominated by $\left(X_{i}\right)_{i}$. We also have that for any scalar $\lambda,\left(T-\lambda i_{\left[\left(y_{i}\right)\right] \rightarrow X}\right)\left(y_{k}\right)=\underline{x}_{n_{k}}-\lambda y_{k}$. But since $\left(y_{k}\right)$ and $\left(\underline{x}_{k}\right)$ are weakly null and $\left(x_{n_{k}}-\lambda y_{k}\right)$ is not norm null, $T-\lambda i_{\left[\left(y_{i}\right)\right] \rightarrow X}$ is not compact. In other words $T$ is not a compact perturbation of a scalar multiple of the inclusion. 
Proof of Lemma 3.4. Since $\ell_{1}$ does not embed in $X$, by Rosenthal's $\ell_{1}$ Theorem [18] and a diagonal argument, by passing to subsequences of $\left(x_{i}\right),\left(x_{i}^{n}\right)$ and $\left(z_{i}\right)$ and relabeling we can assume that $\left(x_{i}\right)$ and $\left(x_{i}^{n}\right)_{i}$ are weakly Cauchy for all $n \in \mathbb{N}$. Set $\left(\underline{x}_{i}\right)_{i}=\left(x_{2 i}-x_{2 i-1}\right)_{i}$, $\left(\underline{x}_{i}^{n}\right)_{i}=\left(x_{2 i}^{n}-x_{2 i-1}^{n}\right)_{i}$ and $\left(\underline{z}_{i}\right)_{i}=\left(z_{2 i}-z_{2 i-1}\right)_{i}$. For every $n \in \mathbb{N}$ the sequences $\left(\underline{x}_{i}\right)$ and $\left(\underline{x}_{i}^{n}\right)_{i}$ are weakly null. Moreover the sequences $\left(\underline{x}_{i}\right)_{i}$ and $\left(\underline{z}_{i}\right)_{i}$ satisfy (11) and the sequences $\left(\underline{x}_{i}^{n}\right)_{i}$ and $\left(\underline{z}_{i}\right)$ satisfy $(12)$ of Theorem 3.2.

Since $\left(\underline{x}_{i}\right)$ and $\left(\underline{x}_{i}^{n}\right)_{i}$ are weakly null for all $n \in \mathbb{N}$, and satisfy conditions (11) and (12) of Theorem 3.2, we have that the spreading models of normalized weakly null sequences in $X$ are not uniformly equivalent. Thus, (since every normalized suppression 1-unconditional basic sequence 2-dominates the unit vector basis of $c_{0}$ ), for every $n \in \mathbb{N}$, there exists a normalized weakly null sequence $\left(u_{i}^{n}\right)_{i}$ in $X$ which has spreading model $\left(\tilde{u}_{i}^{n}\right)_{i}$ and there exist finitely supported scalars $\left(a_{i}^{(n)}\right)_{i}$ such that $\left\|\sum_{i} a_{i}^{(n)} \tilde{u}_{i}^{n}\right\| \geq 2^{2 n}$ yet $\max _{i}\left|a_{i}^{(n)}\right|=1$. By $[2$, Proposition 3.2] there exists a seminormalized weakly null sequence $\left(u_{i}\right)$ in $X$ having spreading model $\left(\tilde{u}_{i}\right)$ such that $\left(\tilde{u}_{i}\right) 2^{n}$-dominates $\left(\tilde{u}_{i}^{n}\right)_{i}$. Thus $\left\|\sum_{i} a_{i}^{(n)} \tilde{u}_{i}\right\| \geq \frac{1}{2^{n}}\left\|\sum_{i} a_{i}^{(n)} \tilde{u}_{i}^{n}\right\| \geq 2^{n}$. Hence $\left(\tilde{u}_{i}\right)$ is not equivalent to the unit vector basis of $c_{0}$. By passing to a subsequence of $\left(u_{i}\right)$ and relabeling we can assume by Theorem 1.1 that $\left(u_{i}\right)$ is Schreier unconditional. Moreover, by passing to a subsequence of $\left(u_{i}\right)$ and relabeling we can assume that for any Schreier set $F \subseteq \mathbb{N}$ and scalars $\left(a_{i}\right)_{i \in F}$,

$$
\frac{1}{2}\left\|\sum_{i \in F} a_{i} \tilde{u}_{i}\right\| \leq\left\|\sum_{i \in F} a_{i} u_{i}\right\| \leq 2\left\|\sum_{i \in F} a_{i} \tilde{u}_{i}\right\| .
$$

Define the basic sequence $\left(Z_{i}\right)$ by

$$
\left\|\sum a_{i} Z_{i}\right\|:=\max \left(\left\|\sum a_{i} \underline{z}_{i}\right\|,\left\|\sum a_{i} u_{i}\right\|\right),
$$

(first for finitely supported sequences $\left(a_{i}\right)$ and then take its completion). Obviously $\left(Z_{i}\right)$ has a spreading model $\left(\tilde{Z}_{i}\right)$ which satisfies

$$
\left\|\sum a_{i} \tilde{Z}_{i}\right\|=\max \left(\left\|\sum a_{i} \underline{\tilde{z}}_{i}\right\|,\left\|\sum a_{i} \tilde{u}_{i}\right\|\right),
$$

for finitely supported sequences of scalars $\left(a_{i}\right)$.

Since $\left(u_{i}\right)$ and $\left(\underline{x}_{i}^{n}\right)_{i}$ are weakly null seminormalized sequences in $X$ for all $n \in \mathbb{N}$, by [2, Proposition 3.2] there exist weakly null basic sequences $\left(X_{i}^{n}\right)_{i}$ in $X$ such that $0<$ $\inf _{n, i}\left\|X_{i}^{n}\right\| \leq \sup _{n, i}\left\|X_{i}^{n}\right\|<\infty$ and the spreading model $\left(\tilde{X}_{i}^{n}\right)_{i}$ of $\left(X_{i}^{n}\right)_{i} 1$-dominates the spreading models of both sequences $\left(\underline{x}_{i}^{n}\right)_{i}$ and $\left(u_{i}\right)$. By the definitions of $\left(X_{i}^{n}\right)_{i}$ and $\left(Z_{i}\right)$ it is obvious that $\left(X_{i}^{n}\right)_{i}$ and $\left(Z_{i}\right)$ satisfy condition (12) of Theorem 3.2. Thus we have satisfied conditions $(\mathrm{d})$ and $(\mathrm{e})$ of Lemma 3.4. It remains to define the basic sequence $\left(X_{i}\right)$ in order to satisfy (a), (b) and (c).

Before defining $\left(X_{i}\right)$ we define an auxiliary basic sequence $\left(u_{i}^{-}\right)$by

$$
\left\|\sum_{i} a_{i} u_{i}^{-}\right\|:=\sup _{1 \leq n<m} \frac{1}{\sqrt{L_{m-n}}}\left\|\sum_{i=n}^{m-1} a_{i} u_{i}\right\|,
$$

where

$$
L_{k}:=\left\|\sum_{i=1}^{k} u_{i}\right\|
$$


(first for finitely supported sequences $\left(a_{i}\right)$ of scalars and then take the completion). Obviously $\left(u_{i}^{-}\right)$is seminormalized since

$$
\frac{\inf _{j}\left\|u_{j}\right\|}{\sqrt{L_{1}}} \leq\left\|u_{i}^{-}\right\| \leq \frac{\sup _{j}\left\|u_{j}\right\|}{\inf _{j} \sqrt{L_{j}}}, \text { for all } i
$$

Notice that

$$
\begin{aligned}
\left\|\sum_{i=1}^{n} u_{i}^{-}\right\| & \geq \frac{\left\|\sum_{i=1}^{n} u_{i}\right\|}{\sqrt{L_{n}}}=\frac{L_{n}}{\sqrt{L_{n}}}=\sqrt{L_{n}} \\
& \geq \sqrt{\left.\frac{1}{1+C}\left\|\sum_{i=\lceil n / 2\rceil}^{n} u_{i}\right\| \text { (where } C \text { is the basis constant of }\left(u_{i}\right)\right)} \\
& \geq \frac{1}{2} \sqrt{\frac{1}{1+C}\left\|\sum_{i=\lceil n / 2\rceil}^{n} \tilde{u}_{i}\right\|}(\text { by }(15)),
\end{aligned}
$$

where the last quantity tends to infinity as $n \rightarrow \infty$ since the basic sequence $\left(\tilde{u}_{i}\right)$ is spreading, unconditional and not equivalent to the unit vector basis of $c_{0}$. Thus

$$
\left\|\sum_{i=1}^{n} u_{i}^{-}\right\| \rightarrow \infty \text { as } n \rightarrow \infty .
$$

Claim 1: $\left(u_{i}^{-}\right)<<\left(u_{i}\right)$.

In order to prove Claim 1 , let $0<\varepsilon<1$ and choose $N \in \mathbb{N}$ such that $\sqrt{L_{k}}>2 C / \varepsilon$ for all $k>N$ (by (16)) where $C$ is the basis constant of $\left(u_{i}\right)$. Let

$$
\delta:=\min _{1 \leq n \leq N} \frac{\varepsilon \sqrt{L_{n}}}{\sup _{i}\left\|u_{i}\right\| n} .
$$

Let $\left(a_{i}\right)$ be a finitely supported sequence of scalars such that $\left\|\sum a_{i} u_{i}^{-}\right\|=1$ and $\left|a_{i}\right| \leq \delta$. Let $1 \leq n_{0}<m_{0}$ such that

$$
1=\left\|\sum a_{i} u_{i}^{-}\right\|=\frac{1}{\sqrt{L_{m_{0}-n_{0}}}}\left\|\sum_{i=n_{0}}^{m_{0}-1} a_{i} u_{i}\right\| .
$$

Notice that if $m_{0}-n_{0} \leq N$ then

$$
\begin{aligned}
\frac{1}{\sqrt{L_{m_{0}-n_{0}}}\left\|\sum_{i=n_{0}}^{m_{0}-1} a_{i} u_{i}\right\|} & \leq \frac{1}{\sqrt{L_{m_{0}-n_{0}}}}\left(m_{0}-n_{0}\right) \max _{n_{0} \leq i \leq m_{0}-1}\left|a_{i}\right| \sup _{i}\left\|u_{i}\right\| \\
& \leq \frac{1}{\sqrt{L_{m_{0}-n_{0}}}}\left(m_{0}-n_{0}\right) \delta \sup _{i}\left\|u_{i}\right\| \\
& \leq \frac{1}{\sqrt{L_{m_{0}-n_{0}}}}\left(m_{0}-n_{0}\right) \frac{\varepsilon \sqrt{L_{m_{0}-n_{0}}}}{\sup _{i}\left\|u_{i}\right\|\left(m_{0}-n_{0}\right)} \sup _{i}\left\|u_{i}\right\| \\
& =\varepsilon<1,
\end{aligned}
$$


which contradicts (17). Thus $m_{0}-n_{0}>N$. Therefore, if $C$ is the basis constant of $\left(u_{i}\right)$, equation (17) implies that

$$
\left\|\sum a_{i} u_{i}\right\| \geq \frac{1}{2 C}\left\|\sum_{i=n_{0}}^{m_{0}-1} a_{i} u_{i}\right\| \geq \frac{1}{2 C} \sqrt{L_{m_{0}-n_{0}}}>\frac{1}{2 C} \frac{2 C}{\varepsilon}=\frac{1}{\varepsilon},
$$

(by the choice of $N$ and the fact that $m_{0}-n_{0}>N$ ). This finishes the proof of Claim 1 .

Since $\left(u_{i}\right)$ is Schreier unconditional, we easily obtain that $\left(u_{i}^{-}\right)$is Schreier unconditional. Also it is easy to verify that $\left(u_{i}^{-}\right)$has a spreading model $\left(\tilde{u}_{i}^{-}\right)$which satisfies

$$
\left\|\sum a_{i} \tilde{u}_{i}^{-}\right\|=\sup _{1 \leq n<m} \frac{1}{\sqrt{L_{m-n}}}\left\|\sum_{i=n}^{m-1} a_{i} \tilde{u}_{i}\right\|,
$$

for all finitely supported sequences $\left(a_{i}\right)$ of scalars. Thus by $(15)$ we obtain that for any Schreier set $F \subseteq \mathbb{N}$ and scalars $\left(a_{i}\right)_{i \in F}$,

$$
\frac{1}{2}\left\|\sum_{i \in F} a_{i} \tilde{u}_{i}^{-}\right\| \leq\left\|\sum_{i \in F} a_{i} u_{i}^{-}\right\| \leq 2\left\|\sum_{i \in F} a_{i} \tilde{u}_{i}^{-}\right\| .
$$

Also, since $\left(\tilde{u}_{i}\right)$ is unconditional we obtain that $\left(\tilde{u}_{i}^{-}\right)$is unconditional. Since $\left(\tilde{u}_{i}^{-}\right)$is spreading, unconditional and it is not equivalent to the unit vector basis of $c_{0}$ (by (16)), it is easy to see that $\left(\tilde{u}_{i}^{-}\right)$has Property P.

Claim 2: $\left(u_{i}^{-}\right)$has Property P.

This will follow from the fact that $\left(\tilde{u}_{i}^{-}\right)$has Property $\mathrm{P}$, equation $(18)$ and that $\left(u_{i}^{-}\right)$is Schreier unconditional. Indeed, let $\rho>0$. Since $\left(\tilde{u}_{i}^{-}\right)$has Property $\mathrm{P}$, there exists $M \in \mathbb{N}$ such that if $\left\|\sum a_{i} \tilde{u}_{i}\right\| \leq 1$ then $\left|\left\{i:\left|a_{i}\right|>\frac{\rho}{2 C_{S}}\right\}\right| \leq M$ (where $C_{S}$ is the Schreier unconditionality constant of $\left(u_{i}^{-}\right)$). Assume that $\left\|\sum b_{i} u_{i}^{-}\right\| \leq 1$ and let $A=\left\{i:\left|b_{i}\right|>\rho\right\}$. We claim that $|A| \leq 3 M$ which finishes the proof of Claim 2. Indeed, if $|A|>3 M$ then let $A_{1}, A_{2} \subseteq A$ with $A_{1} \cup A_{2}=A, a_{1}<a_{2}$ for all $a_{1} \in A_{1}, a_{2} \in A_{2}$ and $\left|A_{2}\right|=\lfloor|A| / 2\rfloor$. Since $|A|>3 M$ we have that

$$
\left|A_{2}\right|>M \text {. }
$$

Since $A_{2}$ is a Schreier set we obtain by (18),

$$
\left\|\sum_{i \in A_{2}} b_{i} \tilde{u}_{i}^{-}\right\| \leq 2\left\|\sum_{i \in A_{2}} b_{i} u_{i}^{-}\right\| \leq 2 C_{S}\left\|\sum b_{i} u_{i}^{-}\right\| \leq 2 C_{S}
$$

Therefore

$$
\left\|\sum_{i \in A_{2}} \frac{b_{i}}{2 C_{S}} \tilde{u}_{i}^{-}\right\| \leq 1
$$

Hence $\left|\left\{i \in A_{2}:\left|\frac{b_{i}}{2 C_{S}}\right|>\frac{\rho}{2 C_{S}}\right\}\right| \leq M$ i.e. $\left|A_{2}\right| \leq M$ which contradicts (19). This finishes the proof of Claim 2.

Now define the basic sequence $\left(X_{i}\right)$ by

$$
\left\|\sum a_{i} X_{i}\right\|:=\max \left(\left\|\sum a_{i} \underline{x}_{i}\right\|,\left\|\sum a_{i} u_{i}^{-}\right\|\right),
$$

(first for finitely supported sequences $\left(a_{i}\right)$ of scalars and then take the completion). Obviously $\left(X_{i}\right)$ dominates $\left(\underline{x}_{i}\right)$ thus $(\mathrm{a})$ is satisfied. Since $\left(u_{i}^{-}\right)$has Property $\mathrm{P}$ we obtain that $\left(X_{i}\right)$ has 
Property P, hence (c) is satisfied. Also, since $\left(\underline{x}_{i}\right)<<\left(\underline{z}_{i}\right)$ and $\left(u_{i}^{-}\right)<<\left(u_{i}\right)$ we have that $\left(X_{i}\right)<<\left(Z_{i}\right)$, therefore (b) is satisfied.

Now we present the proof of Lemma 3.5. Our arguments resemble the ones found in [2].

Proof of Lemma 3.5. Since $\left(X_{n}\right)$ has Property P, for each $\rho>0$ we can define $M=M(\rho)$ such that if $\left\|\sum a_{i} X_{i}\right\|=1$ then $\left|\left\{i:\left|a_{i}\right|>\rho\right\}\right| \leq M$.

Let $\left(\varepsilon_{j}\right)_{j=1}^{\infty}$ be such that

$$
\sum_{j=2}^{\infty} \frac{\varepsilon_{j-1}}{\delta_{j}} \leq \frac{1}{2}
$$

Since $\left(Z_{n}\right)>>\left(\tilde{X}_{n}\right)$ by $(10)$ we may choose a decreasing sequence $\left(\rho_{j}\right)_{j=1}^{\infty} \subseteq(0,1]$ such that $\sum_{j} \sqrt{\rho_{j}}(j+1) \leq 1 / 4$ and satisfying the following: for all $\left(a_{i}\right) \in c_{00}$ with $\left|a_{i}\right| \in\left[0, \sqrt{\rho_{j}}\right]$ for each $i$ and $\left\|\sum a_{i} \tilde{x}_{i}\right\|=1$ we have

$$
\left\|\sum a_{i} \tilde{X}_{i}\right\| \leq \varepsilon_{j}\left\|\sum a_{i} Z_{i}\right\|
$$

Finally let $M_{j}=M\left(\rho_{j}\right)$ as above.

By the definition of spreading models, by passing to a subsequence of $\left(X_{i}\right)$ and relabeling, we can assume that if $j \leq F$ and $|F| \leq M_{j}$ then for all $\left(a_{i}\right) \in c_{00}$,

$$
\frac{1}{2}\left\|\sum_{i \in F} a_{i} X_{i}\right\| \leq\left\|\sum_{i \in F} a_{i} \tilde{X}_{i}\right\| \leq 2\left\|\sum_{i \in F} a_{i} X_{i}\right\| .
$$

Now fix $\left(a_{i}\right) \in c_{00}$ such that $\left\|\sum a_{i} X_{i}\right\|=1$. For $j \in \mathbb{N}$ consider the vector $\tilde{y}=$ $\sum_{i>j ; \rho_{j}<\left|a_{i}\right| \leq \rho_{j-1}} a_{i} \tilde{X}_{i}$. If $\|\tilde{y}\| \geq \sqrt{\rho_{j-1}}$ then

$$
\begin{aligned}
\|\tilde{y}\| & =\|\tilde{y}\|\left\|\frac{\tilde{y}}{\|\tilde{y}\|}\right\|=\|\tilde{y}\|\left\|\sum_{\substack{i>j \\
\rho_{j}<\left|a_{i}\right| \leq \rho_{j-1}}} \frac{a_{i}}{\|\tilde{y}\|} \tilde{X}_{i}\right\| \\
& \left.\leq\|\tilde{y}\| \varepsilon_{j-1}\left\|\sum_{\substack{i>j \\
\rho_{j}<\left|a_{i}\right| \leq \rho_{j-1}}} \frac{a_{i}}{\|\tilde{y}\|} Z_{i}\right\| \text { (by (20) since }\left\|\sum_{\substack{i>j \\
\rho_{j}<\left|a_{i}\right| \leq \rho_{j-1}}} \frac{a_{i}}{\|\tilde{y}\|} \tilde{X}_{i}\right\|=1\right) \\
& =\varepsilon_{j-1}\left\|\sum_{\substack{i>j \\
\rho_{j}<\left|a_{i}\right| \leq \rho_{j-1}}} a_{i} Z_{i}\right\| .
\end{aligned}
$$

Thus in general, (without assuming that $\|\tilde{y}\| \geq \sqrt{\rho_{j-1}}$ ), we get

$$
\|\tilde{y}\| \leq \sqrt{\rho_{j-1}}+\varepsilon_{j-1}\left\|\sum_{i>j ; \rho_{j}<\left|a_{i}\right| \leq \rho_{j-1}} a_{i} Z_{i}\right\| .
$$


Let $\rho_{0}$ be twice the basis constant of $\left(X_{i}\right)$ divided by the $\inf _{i}\left\|X_{i}\right\|$. Since $\left\|\sum a_{i} X_{i}\right\|=1$, we have that $\left|a_{i}\right| \leq \rho_{0}$.

$$
\begin{aligned}
1 & =\left\|\sum a_{i} X_{i}\right\| \leq \sum_{j=1}^{\infty}\left\|\sum_{\rho_{j}<\left|a_{i}\right| \leq \rho_{j-1}} a_{i} X_{i}\right\| \\
& \leq\left\|\sum_{\rho_{1}<\left|a_{i}\right| \leq \rho_{0}} a_{i} X_{i}\right\|+\sum_{j=2}^{\infty}\left\|\sum_{i \leq j ; \rho_{j}<\left|a_{i}\right| \leq \rho_{j-1}} a_{i} X_{i}\right\|+\sum_{j=2}^{\infty}\left\|\sum_{i>j ; \rho_{j}<\left|a_{i}\right| \leq \rho_{j-1}} a_{i} X_{i}\right\| \\
& \leq \sup _{F \subseteq \mathbb{N},|F| \leq M_{1}} \delta_{1}\left\|\sum_{i \in F} a_{i} Z_{i}\right\|+\sum_{j=2}^{\infty} j \rho_{j-1}+2 \sum_{j=2}^{\infty}\left\|\sum_{i>j ; \rho_{j}<\left|a_{i}\right| \leq \rho_{j-1}} a_{i} \tilde{X}_{i}\right\|
\end{aligned}
$$

where

$$
\delta_{1}=\sup \left\{\frac{\left\|\sum_{i \in F} a_{i} X_{i}\right\|}{\left\|\sum_{i \in F} a_{i} Z_{i}\right\|}:\left(a_{i}\right)_{i \in F} \subseteq \mathbb{C} \text { with }|F| \leq M_{1} \text { and }\left(a_{i}\right)_{i \in F} \neq 0^{F}\right\}
$$

which is clearly finite by using an $\ell_{1}$ estimate for the numerator and an $\ell_{\infty}$ estimate for the denominator. Note the third piece of the last inequality is true by (21) since the cardinality of $\left\{i>j: \rho_{j}<\left|a_{i}\right| \leq \rho_{j-1}\right\}$ is at most $M_{j}$. Continuing the calculations from above, we get

$$
\begin{aligned}
1 & \leq \sup _{F \subseteq \mathbb{N},|F| \leq M_{1}} \delta_{1}\left\|\sum_{i \in F} a_{i} Z_{i}\right\|+\frac{1}{4}+2 \sum_{j=2}^{\infty} \sqrt{\rho_{j-1}}+2 \sum_{j=2}^{\infty} \varepsilon_{j-1}\left\|\sum_{i>j ; \rho_{j}<\left|a_{i}\right| \leq \rho_{j-1}} a_{i} Z_{i}\right\| \text { by }(22) \\
& \leq \sup _{F \subseteq \mathbb{N},|F| \leq M_{1}} \delta_{1}\left\|\sum_{i \in F} a_{i} Z_{i}\right\|+\frac{1}{2}+2 \sum_{j=2}^{\infty} \frac{\varepsilon_{j-1}}{\delta_{j}} \delta_{j}\left\|\sum_{i>j ; \rho_{j}<\left|a_{i}\right| \leq \rho_{j-1}} a_{i} Z_{i}\right\| \\
& \leq \sup _{F \subseteq \mathbb{N},|F| \leq M_{1}} \delta_{1}\left\|\sum_{i \in F} a_{i} Z_{i}\right\|+\frac{1}{2}+\sup _{n \geq 2} \sup _{n \leq F \subseteq \mathbb{N} ;|F| \leq M_{n}} \delta_{n}\left\|\sum_{i \in F} a_{i} Z_{i}\right\| .
\end{aligned}
$$

Thus

$$
1 \leq 2 \sup _{n \in \mathbb{N}} \sup _{n \leq F \subseteq \mathbb{N} ;|F| \leq M_{n}} \delta_{n}\left\|\sum_{i \in F} a_{i} Z_{i}\right\|
$$

proving the lemma.

Now we present the proof of Lemma 3.6.

Proof of Lemma 3.6. Assume that for each $n \in \mathbb{N}\left(X_{j}^{M_{n}}\right)_{j}$ has spreading model $\left(\tilde{X}_{j}^{M_{n}}\right)_{j=1}^{M_{n}}$, $\left(\tilde{X}_{j}^{M_{n}}\right)_{j} C$-dominates $\left(Z_{i}\right)_{i=1}^{M_{n}}$ and moreover if $|F| \leq M_{n}$ and $\left(a_{j}\right)_{j \in F}$ are scalars then

$$
\frac{1}{2}\left\|\sum_{j \in F} a_{j} \tilde{X}_{j}^{M_{n}}\right\| \leq\left\|\sum_{j \in F} a_{j} X_{j}^{M_{n}}\right\| \leq 2\left\|\sum_{j \in F} a_{j} \tilde{X}_{j}^{M_{n}}\right\| .
$$

By Remark 2.3 by passing to subsequences and relabeling, assume that $\left(X_{j}^{M_{n}}\right)_{(n, j) \in I}$ forms a regular array with basis constant at most equal to 2. Apply Theorem 1.2 to $\left(X_{j}^{M_{n}}\right)_{(n, j) \in I}$ to get a subarray which satisfies the conclusion of Theorem 1.2. By relabeling call $\left(X_{j}^{M_{n}}\right)_{(n, j) \in I}$ the resulting subarray. Define 


$$
y_{j}=\sum_{n=1}^{j} \delta_{n} X_{\ell_{j}}^{M_{n}}
$$

where $\left(\ell_{j}\right)$ is an increasing sequence of positive integers which guarantees that $\left|x_{i}^{*} y_{i}\right|<\varepsilon$. Note that $\left(y_{j}\right)$ is weakly null since $\left(X_{j}^{M_{n}}\right)_{j}$ is weakly null for all $n$ and $\delta_{n}$ is summable. Since $\left(X_{j}^{M_{n}}\right)_{(n, j) \in I}$ is regular, $\left(y_{j}\right)$ is a basic sequence with

$$
\left\|y_{j}\right\| \geq \frac{\delta_{1}}{2} \inf _{n, m}\left\|X_{m}^{n}\right\|
$$

( since the basis constant of $\left(X_{j}^{M_{n}}\right)_{(n, j) \in I}$ is at most equal to 2 by Remark 2.3). Since $\left(\delta_{n}\right)$ is summable, $\left(y_{j}\right)$ is also bounded. Fix $n \in \mathbb{N}$ and let $n \leq F \subseteq \mathbb{N}$, with $|F| \leq M_{n}$. Then

$$
\begin{aligned}
\left\|\sum a_{j} y_{j}\right\| & \geq \frac{1}{3}\left\|\sum_{j \in F ; j \geq n} \delta_{n} a_{j} X_{\ell_{j}}^{M_{n}}\right\| \text { (by Theorem 1.2) } \\
& \geq \frac{1}{6}\left\|\sum_{j \in F ; j \geq n} \delta_{n} a_{j} \tilde{X}_{j}^{M_{n}}\right\|(\text { by }(23)) \\
& =\frac{1}{6}\left\|\sum_{j \in F ; j \geq n} \delta_{n} a_{j} \tilde{X}_{k_{j}}^{M_{n}}\right\|
\end{aligned}
$$

where the map $F \ni j \mapsto k_{j} \in\{1,2, \ldots,|F|\}$ is a $1-1$ increasing function

$$
\geq \frac{1}{6 C} \delta_{n}\left\|\sum_{j \in F ; j \geq n} a_{j} Z_{j}\right\|(\text { by }(12))
$$

Thus

$$
\left\|\sum a_{j} y_{j}\right\| \geq \sup _{n} \frac{1}{6 C} \sup _{n \leq F \subseteq \mathbb{N} ;|F| \leq M_{n}} \delta_{n}\left\|\sum_{i \in F} a_{i} Z_{i}\right\| .
$$

An easy corollary of Theorem 3.2 is Theorem 3.8 which is obtained if we set $\left(z_{i}\right)$ to be the unit vector basis of $\ell_{p}$ for some fixed $p \in[1, \infty)$. In order to formulate Theorem 3.8 we need to recall the notion of the Krivine set of a 1-subsymmetric (i.e. 1-equivalent to all of its subsequences and unconditional) basic sequence.

Definition 3.7. Let $\left(x_{n}\right)$ be a 1-subsymmetric basic sequence in some Banach space. The Krivine set of $\left(x_{n}\right)$ is defined to be the set of all $p$ 's in $[1, \infty]$ with the following property. For all $\varepsilon>0$ and $N \in \mathbb{N}$ there exists $m \in \mathbb{N}$ and scalars $\left(\lambda_{k}\right)_{k=1}^{m}$ such that for all scalars $\left(a_{n}\right)_{n=1}^{N}$,

$$
\frac{1}{1+\varepsilon}\left\|\left(a_{n}\right)_{n=1}^{N}\right\|_{p} \leq\left\|\sum_{n=1}^{N} a_{n} y_{n}\right\| \leq(1+\varepsilon)\left\|\left(a_{n}\right)_{n=1}^{N}\right\|_{p}
$$

where

$$
y_{n}=\sum_{k=1}^{m} \lambda_{k} x_{(n-1) m+k} \text { for } n=1, \ldots, N
$$

and $\|\cdot\|_{p}$ denotes the norm of the space $\ell_{p}$. 
The Krivine's theorem as it was proved by H. Rosenthal [17] and H. Lemberg [13] states that if $\left(x_{n}\right)$ is a 1-subsymmetric basic sequence then the Krivine set of $\left(x_{n}\right)$ is non-empty. In particular, if $\left(x_{n}\right)$ is a seminormalized weakly null basic sequence in a Banach space having spreading model $\left(\tilde{x}_{n}\right)$ then $\left(\tilde{x}_{n}\right)$ is 1-subsymmetric, hence the Krivine set of $\left(\tilde{x}_{n}\right)$ is non-empty.

Theorem 3.8. Let $X$ be a Banach space. Assume that there exist seminormalized basic sequences $\left(x_{i}\right),\left(y_{i}\right)$ in $X$ such that $\left(x_{i}\right)$ has spreading model $\left(\tilde{x}_{i}\right)$ which is s.c. dominated by the unit vector basis of $\ell_{p}$, for some $p \in[1, \infty)$ and $\left(y_{i}\right)$ has spreading model $\left(\tilde{y}_{i}\right)$ which is unconditional and $p$ belongs to the Krivine set of $\left(\tilde{y}_{i}\right)$. Then there exists a subspace $Y$ of $X$ with a basis and an operator $T \in \mathcal{L}(Y, X)$ which is not a compact perturbation of a multiple of the inclusion map.

For applying this result for $p=1$, recall that by [2, Proposition 2.1], a seminormalized subsymmetric basic sequence is not equivalent to the unit vector basis of $\ell_{1}$ if and only if it is s.c. dominated by the unit vector basis of $\ell_{1}$. Thus Theorem 3.8 for $p=1$ implies $[2$, Theorem 6.4].

Proof of Theorem 3.8. Since $p$ belongs to the Krivine set of $\left(\tilde{y}_{i}\right)$ then for all $n \in \mathbb{N}$ there exists $\left(x_{i}^{n}\right)_{i \in \mathbb{N}}$ a block sequence of $\left(y_{i}\right)$ of identically distributed blocks such that any $n$ terms of $\left(x_{i}^{n}\right)_{i \in \mathbb{N}}$ are 2-equivalent to the unit vector basis of $\ell_{p}^{n}$. Then apply Theorem 3.2 for $\left(z_{i}\right)$ being the unit vector basis of $\ell_{p}$.

\section{An Application of Theorem 3.8}

Next we give an application of Theorem 3.8. As mentioned before the "multiple of the inclusion plus compact" problem is non trivial only in HI-saturated Banach spaces. The HI space to which Theorem 3.8 will be applied was constructed by N. Dew [7], and here will be denoted by $D$. The construction of the space $D$ is based on the 2-convexification of the Schlumprecht space $S[20]$ in a similar manner that the space of T.W. Gowers and B. Maurey [11] is based on $S$. We recall the necessary definitions.

Let $X$ be a Banach space with a basis $\left(e_{i}\right)$. For any interval $E$ in $\mathbb{N}$ and a vector $x=$ $\sum x_{j} e_{j} \in X$ define $E x=\sum_{j \in E} x_{j} e_{j} \in X$. There is a unique norm $\|\cdot\|_{S}$ on $c_{00}$ which satisfies:

$$
\|x\|_{S}=\sup \left\{\frac{1}{f(\ell)} \sum_{i=1}^{\ell}\left\|E_{i} x\right\|_{S}: E_{1}<\cdots<E_{\ell}\right\} \vee\|x\|_{\ell_{\infty}}
$$

where $f(\ell)=\log _{2}(\ell+1)$. The completion of $c_{00}$ under this norm is the Banach space $S$. Let $S_{2}$ be its 2-convexification. Recall if $X$ is a Banach space having an unconditional basis $\left(e_{n}\right)$, then we can define the 2-convexification $X_{2}$ of $X$ by the norm

$$
\left\|\sum a_{n} \sqrt{e_{n}}\right\|_{X_{2}}:=\left(\left\|\sum a_{n}^{2} e_{n}\right\|_{X}\right)^{1 / 2}
$$

where $\left(\sqrt{e_{n}}\right)$ denotes the basis of $X_{2}$, (we will talk more later about the "square root" map from $X$ to $X_{2}$ ). We will show that the spreading model of the unit vector basis of $D$ is the unit vector basis of $S_{2}$. Before we do this we must see the definition of $D$.

In order to define the Banach space $D$, a lacunary set $J \subseteq \mathbb{N}$ is used which has the property that if $n, m \in J$ and $n<m$ then $8 n^{4} \leq \log \log \log m$, and $f(\min J) \geq 45^{4}$. Write $J$ in increasing order as $\left\{j_{1}, j_{2}, \ldots\right\}$. Now let $K \subset J$ be the set $\left\{j_{1}, j_{3}, j_{5}, \cdots\right\}$ and $L \subset J$ 
be the set $\left\{j_{2}, j_{4}, j_{6}, \ldots\right\}$. Let $\mathbf{Q}$ be the set of scalar sequences with finite support and rational coordinates whose absolute value is at most one. Let $\sigma$ be an injective function from $\mathbf{Q}$ to $L$ such that if $z_{1}, \ldots, z_{i}$ is such a sequence, then $(1 / 400) f\left(\sigma\left(z_{1}, \ldots, z_{i}\right)^{1 / 40}\right)^{\frac{1}{2}} \geq$ $\# \operatorname{supp}\left(\sum_{j=1}^{i} z_{j}\right)$. Then, recursively, we define a set of functionals of the unit ball of the dual space $D^{*}$ as follows: Let

$$
D_{0}^{*}=\left\{\lambda e_{n}^{*}: n \in \mathbb{N},|\lambda| \leq 1\right\} .
$$

Assume that $D_{k}^{*}$ has been defined. Define the norm $\|\cdot\|_{k}$ on $c_{00}$ by

$$
\|x\|_{k}=\sup \left\{\left|x^{*}(x)\right|: x^{*} \in D_{k}^{*}\right\}
$$

and let $\|\cdot\|_{k}^{*}$ denote its dual norm. Then $D_{k+1}^{*}$ is the set of all functionals of the form $F z^{*}$ where $F \subseteq \mathbb{N}$ is an interval and $z^{*}$ has one of the following three forms:

$$
z^{*}=\sum_{i=1}^{\ell} \alpha_{i} z_{i}^{*}
$$

where $\sum_{i=1}^{\ell}\left|\alpha_{i}\right| \leq 1$ and $z_{i}^{*} \in D_{k}^{*}$ for $i=1, \ldots, \ell$.

$$
z^{*}=\frac{1}{\sqrt{f(\ell)}} \sum_{i=1}^{\ell} \alpha_{i} z_{i}^{*}
$$

where $\sum_{i=1}^{\ell} \alpha_{i}^{2} \leq 1, z_{i}^{*} \in D_{k}^{*}$ for $i=1, \ldots, \ell$, and $z_{1}^{*}<\cdots<z_{\ell}^{*}$.

$$
z^{*}=\frac{1}{\sqrt[4]{f(\ell)}} \sum_{i=1}^{\ell} z_{i}^{*} \text { where } z_{i}^{*}=\frac{1}{\sqrt{f\left(m_{i}\right)}} \sum_{j=1}^{m_{i}} \alpha_{i, j} \frac{E z_{i, j}^{*}}{\left\|E z_{i, j}^{*}\right\|_{k}^{*}}
$$

for certain $\left(\alpha_{i, j}\right)$ where $\sum_{i, j} \alpha_{i, j}^{2} \leq 1\left(\alpha_{i, j}\right.$ 's are explicitly chosen in [7], but the exact values are not needed for our purposes) where $z_{i, j}^{*} \in D_{k}^{*}$ for $1 \leq i \leq \ell$ and $1 \leq j \leq m_{i}$, $z_{1,1}^{*}<\cdots<z_{1, m_{1}}^{*}<z_{2,1}^{*}<\cdots<z_{\ell, m_{\ell}}^{*}, m_{1}=j_{2 \ell}, \beta z_{i}^{*}$ has rational coordinates for some $\beta>0$ (whose exact value is not needed for our purpose), $m_{i+1}=\sigma\left(\beta z_{1}^{*}, \ldots, \beta z_{i}^{*}\right)$, for $i=1, \ldots, \ell-1$ and $E$ is an interval.

Finally, the norm of $D$ is defined by

$$
\|x\|_{D}=\sup \left\{z^{*}(x): z^{*} \in \cup_{k=0}^{\infty} D_{k}^{*}\right\} .
$$

Proposition 4.1. The spreading model of the unit vector basis of $D$ is the unit vector basis of $S_{2}$.

Proof. From the definitions of the two spaces it is easy to see that for $\left(a_{i}\right) \in c_{00},\left\|\sum a_{i} e_{i}\right\|_{S_{2}} \leq$ $\left\|\sum a_{i} e_{i}\right\|_{D}\left(\left(e_{n}\right)\right.$ will denote the bases of both spaces $S_{2}$ and $D$ but there will be no confusion about which space we consider at each moment). Thus to show Proposition 4.1 we need only show for any given $\varepsilon>0$, and finitely many scalars $\left(a_{i}\right)_{i=1}^{N}$ there exists $n_{0}$ such that for any $n_{1}, n_{2}, \ldots, n_{N} \in \mathbb{N}$ with $n_{0}<n_{1}<n_{2}<\cdots<n_{N}$, we have

$$
\|x\|_{D} \leq\|y\|_{S_{2}}+\varepsilon
$$

where $x=\sum_{i=1}^{N} a_{i} e_{n_{i}} \in D$ and $y=\sum_{i=1}^{N} a_{i} e_{i} \in S_{2}$. This will follow immediately once we show by induction on $n$ that for any $n \in \mathbb{N}, \varepsilon>0$ and scalars $\left(a_{i}\right)_{i=1}^{N}$ we have 


$$
\|x\|_{n} \leq\|y\|_{S_{2}}+\varepsilon
$$

where $\|\cdot\|_{n}$ is defined in (25). For " $n=0$ " we have $\|x\|_{0}=\max _{1 \leq i \leq N}\left|a_{i}\right| \leq\|y\|_{S_{2}}$. Now for the inductive step assume that (29) is valid for $n$. Let $\varepsilon>0$ and scalars $\left(a_{i}\right)_{i=1}^{N}$.

First note that by the induction hypothesis there exists $n_{0}^{1} \in \mathbb{N}$ such that for all $n_{0}^{1}<n_{1}<$ $n_{2}<\cdots<n_{N}$ we have

$$
\|x\|_{n} \leq\|y\|_{S_{2}}+\varepsilon .
$$

Secondly, by the inductive hypothesis there exists $n_{0}^{2} \in \mathbb{N}$ such that for all $1 \leq i_{0} \leq j_{0} \leq N$ and $n_{0}^{2}<n_{1}<n_{2}<\cdots<n_{N}$ we have

$$
\left\|\sum_{i=i_{0}}^{j_{0}} a_{i} e_{n_{i}}\right\|_{n} \leq\left\|\sum_{i=i_{0}}^{j_{0}} a_{i} e_{i}\right\|_{S_{2}}+\frac{\varepsilon}{\sqrt{N}}
$$

And finally there exists $j_{0} \in L$ such that $\frac{1}{\sqrt{f\left(j_{0}\right)}} N\|y\|_{S_{2}} \leq \varepsilon$. Let $G=\sigma^{-1}\left(\left\{1,2, \ldots, j_{0}-\right.\right.$ $1\})$, So $G$ is a finite subset of finite sequences of vectors with rational coefficients. Let $n_{0}^{3}$ be the maximum of the support of any vector in any sequence in $G$. And then set $n_{0}=\max \left\{n_{0}^{1}, n_{0}^{2}, n_{0}^{3}\right\}$. Recall the norming vectors $z^{*} \in D_{n+1}^{*}$ can be one of three different types. Each type of functional will present us with a different case.

CASE 1: Let $z^{*}$ be given by (26). Then

$$
\begin{aligned}
\left|z^{*}(x)\right| & \leq \sum_{i=1}^{\ell}\left|\alpha_{i}\right|\left|z_{i}^{*}(x)\right| \\
& \left.\leq \sum_{i=1}^{\ell}\left|\alpha_{i}\right|\left(\|x\|_{S_{2}}+\varepsilon\right) \text { (by the }(30)\right) \\
& =\|x\|_{S_{2}}+\varepsilon .
\end{aligned}
$$

CASE 2: Let $z^{*}$ be given by (27). Thus for $n_{0}<n_{1}<n_{2}<\cdots<n_{N}$ we have

$$
\begin{aligned}
\left|z^{*}(x)\right| & \leq \frac{1}{\sqrt{f(\ell)}} \sum_{j=1}^{\ell}\left|\alpha_{j}\right|\left|z_{j}^{*}(x)\right| \\
& \leq \frac{1}{\sqrt{f(\ell)}} \sum_{j=1}^{\ell}\left|\alpha_{j}\right|\left|z_{j}^{*}\left(E_{j} x\right)\right|
\end{aligned}
$$

where $E_{j}$ is the smallest interval containing the support of $z_{j}^{*}$ intersected with the support of $x$. Continuing the above calculation we have 


$$
\begin{aligned}
\left|z^{*}(x)\right| & \leq \frac{1}{\sqrt{f(\ell)}} \sum_{j=1}^{\ell}\left|\alpha_{j}\right|\left\|E_{j} x\right\|_{n} \\
& \left.\leq \frac{1}{\sqrt{f(\ell)}} \sum_{j=1}^{\ell}\left|\alpha_{j}\right|\left(\left\|E_{j} x\right\|_{s_{2}}+\frac{\varepsilon}{\sqrt{N}}\right) \text { (by }(31)\right) \\
& \leq \frac{1}{\sqrt{f(\ell)}}\left(\sum_{j=1}^{\ell}\left|\alpha_{j}\right|^{2}\right)^{1 / 2}\left(\sum_{j=1}^{\ell}\left\|E_{j} x\right\|_{s_{2}}^{2}\right)^{1 / 2}+\varepsilon
\end{aligned}
$$

(by Cauchy-Schwarz' inequality since there are at most $N$ many nonempty $E_{j}$ 's ) $\leq\|x\|_{s_{2}}+\varepsilon$.

CASE 3: Let $z^{*}$ be given by (28). We can of course assume $z^{*}(x) \neq 0$ thus let $i_{0}$ be the smallest natural number such that

$$
\cup_{i=1}^{i_{0}} \operatorname{supp}\left(z_{i}^{*}\right) \cap\left\{n_{0}+1, n_{0}+2, \ldots\right\} \neq \emptyset .
$$

Then by the definition of $n_{0}^{3}$ we have that $\sigma\left(\beta z_{1}^{*}, \beta z_{2}^{*}, \ldots, \beta z_{i}^{*}\right)>j_{0}$ for $i_{0}+1 \leq i \leq \ell$. Thus

$$
\begin{aligned}
& \leq \frac{1}{\sqrt[4]{f(\ell)}}\left|\frac{1}{\sqrt{f\left(m_{i_{0}}\right)}} \sum_{j=1}^{m_{i_{0}}} \alpha_{i_{0}, j} z_{i_{0}, j}^{*}(x)\right|+\frac{1}{\sqrt[4]{f(\ell)}} \frac{1}{\sqrt{f\left(m_{i_{0}+1}\right)}} \sum_{j=1}^{m_{i_{0}+1}}\left|\alpha_{i_{0}+1, j} \| z_{i_{0}+1, j}^{*}(x)\right|+\cdots \\
& \leq \frac{1}{\sqrt[4]{f(\ell)}}\|y\|_{S_{2}}+\varepsilon+\frac{1}{\sqrt[4]{f(\ell)}} \frac{1}{\sqrt{f\left(m_{i_{0}+1}\right)}} \sum_{\left\{1 \leq j \leq m_{i_{0}+1} z_{i_{0}+1, j}^{*} x \neq 0\right\}}\left|\alpha_{i_{0}+1, j} \| z_{i_{0}+1, j}^{*}(x)\right|+\cdots \text { (by CASE 2) } \\
& \leq \frac{1}{\sqrt[4]{f(\ell)}}\|y\|_{S_{2}}+\varepsilon+\frac{1}{\sqrt[4]{f(\ell)}} \frac{1}{\sqrt{f\left(j_{0}\right)}} N\left(\|y\|_{S_{2}}+\varepsilon\right),
\end{aligned}
$$

where the last inequality is valid since $\left|\alpha_{i, j}\right| \leq 1,\left|z_{i, j}^{*}(x)\right| \leq\left\|z_{i, j}^{*}\right\|_{n}^{*}\|x\|_{n} \leq\|x\|_{n} \leq\|y\|_{S_{2}}+\varepsilon$, $m_{i} \geq j_{0}$ for all $i_{0}+1 \leq i \leq \ell$, (by the choice of $n_{0}$ ), and there are at most $N$ many indexes $(i, j)$ for which $z_{i, j}^{*}(x) \neq 0$. The last expression in equation (32) is at most equal to $\|y\|_{S_{2}}+3 \varepsilon$ which finishes the proof.

For the following remark we will need a bit more notation. If $X$ is a Banach space having an unconditional basis $\left(e_{n}\right)$ and $X_{2}$ is the 2-convexification of $X$ then for $x=\sum a_{n} e_{n} \in X$ then there is a canonical image of $x$ in $X_{2}$ which we define as $\sqrt{x}=\sum \operatorname{sign}\left(a_{n}\right) \sqrt{\left|a_{n}\right|} \sqrt{e_{n}} \in X_{2}$ (where $\left(\sqrt{e_{n}}\right)$ denotes the basis of $X_{2}$ ).

Remark 4.2. Let $1 \leq p<\infty$. Let $X$ be a Banach space with an unconditional basis $\left(e_{i}\right)_{i}$ and let $\left(\sqrt{e_{i}}\right)_{i}$ be the basis of $X_{2}$. Then $p$ is in the Krivine set of $\left(e_{i}\right)$ if and only if $2 p$ is in the Krivine set of $\left(\sqrt{e_{i}}\right)$.

Proof. Let $n \in \mathbb{N}$ and $\varepsilon>0$ be given. Since $p$ is in the Krivine set of $X$, there exists a block sequence $\left(v_{i}\right)_{i=1}^{n}$ of $\left(e_{i}\right)$ such that for any scalars $\left(a_{i}\right)_{i=1}^{n}$ we have that 


$$
\frac{1}{1+\varepsilon}\left(\sum_{i=1}^{n} a_{i}^{p}\right)^{1 / p} \leq\left\|\sum_{i=1}^{n} a_{i} v_{i}\right\| \leq(1+\varepsilon)\left(\sum_{i=1}^{n} a_{i}^{p}\right)^{\frac{1}{p}}
$$

Let $w_{i}=\sqrt{v_{i}} \in X_{2}$, and scalars $\left(a_{i}\right)_{i=1}^{n}$ then

$$
\left\|\sum_{i=1}^{n} a_{i} w_{i}\right\|_{X_{2}}=\left\|\sum_{i=1}^{n} a_{i}^{2} v_{i}\right\|_{X}^{\frac{1}{2}} \leq(1+\varepsilon)\left(\sum_{i=1}^{n} a_{i}^{2 p}\right)^{\frac{1}{2 p}}
$$

and

$$
\left\|\sum_{i=1}^{n} a_{i} w_{i}\right\|_{X_{2}}=\left\|\sum_{i=1}^{n} a_{i}^{2} v_{i}\right\|_{X}^{\frac{1}{2}} \geq \frac{1}{1+\varepsilon}\left(\sum_{i=1}^{n} a_{i}^{2 p}\right)^{\frac{1}{2 p}}
$$

The proof of the converse is similar.

It is known [20] that the Krivine set of the unit vector basis of $S$ consists of the singleton $\{1\}$. Thus by Remark 4.2 we have:

Remark 4.3. The Krivine set of the unit vector basis of $S_{2}$ consists of the singleton $\{2\}$.

Proposition 4.4. Let $X, Y$ be Banach spaces with unconditional bases, $\left(x_{n}\right)$ be a basic sequence in $X,\left(y_{n}\right)$ be a basic sequence in $Y$ such that $\left(x_{n}\right)>>\left(y_{n}\right)$. Then $\left(\sqrt{x_{n}}\right)>>\left(\sqrt{y_{n}}\right)$ where $\left(\sqrt{x_{n}}\right)$ and $\left(\sqrt{y_{n}}\right)$ are the canonical images of $\left(x_{n}\right)$ and $\left(y_{n}\right)$ in $X_{2}$ and $Y_{2}$ respectively.

Proof. Note that

$$
\liminf _{n \rightarrow \infty} \inf _{A \subseteq \mathbb{N} ;|A|=n}\left\|\sum_{i \in A} \sqrt{x_{i}}\right\|_{X_{2}}=\liminf _{n \rightarrow \infty} \inf _{A \subseteq \mathbb{N} ;|A|=n}\left\|\sum_{i \in A} x_{i}\right\|_{X}^{\frac{1}{2}}=\infty .
$$

Also

$$
\begin{aligned}
\Delta_{\left(\sqrt{x_{n}}\right),\left(\sqrt{y_{n}}\right)}(\varepsilon) & =\sup \left\{\left\|\sum a_{i} \sqrt{y_{i}}\right\|_{Y_{2}}:\left|a_{i}\right| \leq \varepsilon \text { and }\left\|\sum a_{i} \sqrt{x_{i}}\right\|_{X_{2}}=1\right\} \\
& =\left(\sup \left\{\left\|\sum a_{i}^{2} y_{i}\right\|_{Y}:\left|a_{i}\right|^{2} \leq \varepsilon^{2} \text { and }\left\|\sum a_{i}^{2} x_{i}\right\|_{X}=1\right\}\right)^{\frac{1}{2}} \\
& \leq\left(\Delta_{\left(x_{n}\right),\left(y_{n}\right)}\left(\varepsilon^{2}\right)\right)^{\frac{1}{2}} .
\end{aligned}
$$

The result follows immediately from (33) and (34).

It has been shown in [2, Proposition 2.1] that if $\left(e_{n}\right)$ is the unit vector basis of $\ell_{1}$ and $\left(f_{n}\right)$ is a normalized subsymmetric basic sequence which is not equivalent to $\left(e_{n}\right)$ then $\left(e_{n}\right)>>\left(f_{n}\right)$. Thus since the unit vector basis of $S$ is normalized and subsymmetric we have that the unit vector basis of $\ell_{1}$ s.c. dominates the unit vector basis of $S$. Thus Proposition 4.4 gives:

Remark 4.5. The unit vector basis of $\ell_{2}$ s.c. dominates the unit vector basis of $S_{2}$.

Theorem 4.6. There exists an infinite dimensional subspace $Y$ of $D$ having a basis and $T \in \mathcal{L}(Y, D)$ such that $T \notin \mathbb{C} i_{Y \rightarrow D}+\mathcal{K}(Y, D)$.

Proof. We will refer to $\left(s_{i}\right)$ as the unit vector basis of the Schlumprecht space $S$ and $\left(\sqrt{s_{i}}\right)$ as the unit vector basis of $S_{2}$. Then apply Theorem 3.8 for $p=2$ and $\left(x_{i}\right)_{i}=\left(y_{i}\right)_{i}=$ the unit vector basis of $D$. By Proposition 4.1 we have that $\left(\sqrt{s_{i}}\right)$ is the spreading model of the unit vector basis of $D$. By Remark 4.3 we have that 2 is in the Krivine set of $\left(\sqrt{s_{i}}\right)$. By Remark 4.5 we have that the unit vector basis of $\ell_{2}$ s.c. dominates $\left(\sqrt{s_{i}}\right)$. Thus 
by Theorem 3.8 we have that there exists an infinite dimensional subspace $Y$ of $D$ and $T \in \mathcal{L}(Y, D)$ such that $T \notin \mathbb{C} i_{Y \rightarrow D}+\mathcal{K}(Y, D)$.

\section{REFERENCES}

[1] Albiac, F; Kalton, N. Topics in Banach space theory. Graduate Texts in Mathematics, 233. Springer, New York, 2006.

[2] Androulakis, G., Odell, E., Schlumprecht, Th., Tomczak-Jaegermann, N. On the structure of the spreading models of a Banach space. Canad. J. Math. 57 (2005), no. 4, 673-707.

[3] Argyros, S.A, Mercurakis, S., Tsarpalias, A. Convex unconditionality and summability of weakly null sequences, Israel J. Math. 107, (1998) 157-193.

[4] Beauzamy, B., Lapreste, J.-T. Modeles etales des espaces de Banach. Travaux en Cours. Hermann, Paris, 1984.

[5] Brunel, A., Sucheston, L. On J-convexity and some ergodic super-properties of Banach spaces. Trans. Amer. Math. Soc. 204 (1975), 79-90.

[6] Brunel, A., Sucheston, L. On B-convex Banach spaces. Math. Systems Theory 7 (1974), no. 4, $294-$ 299.

[7] Dew, N. Asymptotic Structure of Banach Spaces, Ph.D dissertation, University of Oxford, St. Johns College (2002) (available at http://eprints.maths.ox.ac.uk/49/01/dew.pdf).

[8] Dilworth, S.J., Odell, E., Schlumprecht, Th., A. Zsak. Partial Unconditionality, preprint.

[9] Gowers, W. T. An infinite Ramsey theorem and some Banach-space dichotomies. Ann. of Math. (2) 156 (2002), no. 3, 797-833.

[10] Gowers, W. T. A remark about the scalar-plus-compact problem. Convex geometric analysis (Berkeley, CA, 1996), 111-115, Math. Sci. Res. Inst. Publ., 34, Cambridge Univ. Press, Cambridge, 1999.

[11] Gowers, W. T.; Maurey, B. The unconditional basic sequence problem. J. Amer. Math. Soc. 6 (1993), no. $4,851-874$.

[12] Halbeisen, L.; Odell, E. On asymptotic models in Banach spaces. Israel J. Math. 139 (2004), 253-291.

[13] Lemberg, H. Nouvelle demonstration d'un théorème de J.-L. Krivine sur la finie representation de $\ell_{p}$ dans un espace de Banach. Israel J. Math. 39 (1981), no. 4, 341-348.

[14] Lindenstrauss, J.; Tzafriri, L. Classical Banach spaces. I. Sequence spaces. (1977) Ergebnisse der Mathematik und ihrer Grenzgebiete, Vol. 92.

[15] Maurey, B.; Rosenthal, H. P. Normalized weakly null sequence with no unconditional subsequence, Studia Math. 61 (1977), no. 1, 77-98.

[16] Odell, E. On Schreier Unconditional Sequences. Contemp. Math. 144 (1993), 197-201.

[17] Rosenthal, H. P. On a theorem of J. L. Krivine concerning block finite representability of $\ell_{p}$ in general Banach spaces. J. Funct. Anal. 28 (1978), no. 2, 197-225.

[18] Rosenthal, H. P. A characterization of Banach spaces containing $\ell_{1}$, Proc. Nat. Acad. Sci. USA 71 (1974), 2411-2413.

[19] Rosenthal, H.P. Some remarks concerning unconditional basic sequences, Longhorn Notes, The University of Texas, Texas Functional Analysis seminar, (1982-1983), 15-48.

[20] Schlumprecht, Th. An arbitrarily distortable Banach space. Israel J. Math. 76 (1991), no. 1-2, 81-95.

[21] Schlumprecht, Th. How many operators exist on a Banach space? Trends in Banach spaces and operator theory (Memphis, TN, 2001), 295-333, Contemp. Math., 321, Amer. Math. Soc., Providence, RI, 2003.

Department of Mathematics, University of South Carolina, Columbia, SC 29208.

E-mail address: giorgis@math.sc.edu, sanacory@math.sc.edu 